\title{
Origin of Abelian gauge symmetries in heterotic/F-theory duality
}

\author{
Mirjam Cvetič, ${ }^{a, b}$ Antonella Grassi, ${ }^{c}$ Denis Klevers, ${ }^{d}$ Maximilian Poretschkin ${ }^{a}$ \\ and Peng Song ${ }^{a}$ \\ ${ }^{a}$ Department of Physics and Astronomy, University of Pennsylvania, \\ Philadelphia, PA 19104-6396, U.S.A. \\ ${ }^{b}$ Center for Applied Mathematics and Theoretical Physics, University of Maribor, \\ Maribor, Slovenia \\ ${ }^{c}$ Department of Mathematics, University of Pennsylvania, \\ Philadelphia, PA 19104-6396, U.S.A. \\ ${ }^{d}$ Theory Group, Physics Department, CERN, \\ CH-1211, Geneva 23, Switzerland \\ E-mail: cvetic@cvetic.hep.upenn.edu, grassi@math.upenn.edu, \\ denis.klevers@cern.ch, mporet@sas.upenn.edu, songpeng@sas.upenn.edu
}

ABSTRACT: We study aspects of heterotic/F-theory duality for compactifications with Abelian gauge symmetries. We consider F-theory on general Calabi-Yau manifolds with a rank one Mordell-Weil group of rational sections. By rigorously performing the stable degeneration limit in a class of toric models, we derive both the Calabi-Yau geometry as well as the spectral cover describing the vector bundle in the heterotic dual theory. We carefully investigate the spectral cover employing the group law on the elliptic curve in the heterotic theory. We find in explicit examples that there are three different classes of heterotic duals that have $\mathrm{U}(1)$ factors in their low energy effective theories: split spectral covers describing bundles with $\mathrm{S}(\mathrm{U}(m) \times \mathrm{U}(1))$ structure group, spectral covers containing torsional sections that seem to give rise to bundles with $\mathrm{SU}(m) \times \mathbb{Z}_{k}$ structure group and bundles with purely non-Abelian structure groups having a centralizer in $\mathrm{E}_{8}$ containing a $\mathrm{U}(1)$ factor. In the former two cases, it is required that the elliptic fibration on the heterotic side has a non-trivial Mordell-Weil group. While the number of geometrically massless U(1)'s is determined entirely by geometry on the F-theory side, on the heterotic side the correct number of U(1)'s is found by taking into account a Stückelberg mechanism in the lower-dimensional effective theory. In geometry, this corresponds to the condition that sections in the two half K3 surfaces that arise in the stable degeneration limit of F-theory can be glued together globally.

KEywords: F-Theory, String Duality, Superstrings and Heterotic Strings, M-Theory ARXIV EPRINT: 1511.08208 


\section{Contents}

1 Introduction and summary of results 1

2 Heterotic/F-theory duality and U(1)-factors 4

2.1 Heterotic/F-theory duality in 8D 4

2.1.1 The standard stable degeneration limit 5

$\begin{array}{lll}\text { 2.1.2 Stable degeneration with other elliptic fiber types } & 7\end{array}$

$\begin{array}{lll}2.2 & \text { Constructing } \mathrm{SU}(N) \text { bundles on elliptic curves and fibrations } & 7\end{array}$

2.2.1 Vector bundles with reduced structure groups 9

$\begin{array}{lll}2.3 & \text { Heterotic/F-theory duality in 6D } & 10\end{array}$

2.4 Massless U(1)-factors in heterotic/F-theory duality 11

$\begin{array}{lll}2.4 .1 & \text { The heterotic Stückelberg mechanism } & 13\end{array}$

2.4.2 U(1)-factors from gluing conditions in half K3-fibrations $\quad 14$

3 Dual geometries with toric stable degeneration $\quad 14$

$\begin{array}{ll}3.1 \text { Constructing an elliptically fibered K3 surface } & 14\end{array}$

$\begin{array}{lll}3.2 & \text { Constructing K3 fibrations } & 16\end{array}$

$\begin{array}{ll}3.3 \text { The toric stable degeneration limit } & 18\end{array}$

3.4 Computing the canonical classes of the half K3 surfaces $X_{2}^{ \pm} \quad 20$

4 Examples of heterotic/F-theory duals with U(1)'s 21

4.1 The geometrical set-up: toric hypersurfaces in $\mathbb{P}^{1} \times \mathrm{Bl}_{1} \mathbb{P}^{(1,1,2)} \quad 21$

4.1.1 Engineering gauge symmetry: specialized sections of $-K_{\mathbb{P}^{1} \times \mathrm{Bl}_{1} \mathbb{P}^{(1,1,2)}} \quad 22$

4.1.2 Stable degeneration and the spectral cover polynomial 23

4.1.3 Promotion to elliptically fibered threefolds 24

4.2 U(1)'s arising from U(1) factors in the heterotic structure group 25

4.2.1 Structure group $\mathrm{U}(1) \times \mathrm{U}(1): \mathrm{E}_{7} \times \mathrm{E}_{7} \times \mathrm{U}(1)$ gauge symmetry 26

4.2.2 Structure group $\mathrm{U}(1) \times \mathrm{S}(\mathrm{U}(2) \times \mathrm{U}(1)): \mathrm{E}_{7} \times \mathrm{E}_{6} \times \mathrm{U}(1)$ gauge symmetry 28

4.2.3 Structure group $\mathrm{U}(1) \times(\mathrm{SU}(2) \times \mathrm{SU}(2) \times \mathrm{U}(1)): \mathrm{E}_{7} \times \mathrm{SO}(9) \times \mathrm{U}(1)$ gauge symmetry 30

4.2.4 Example with only one massive $\mathrm{U}(1)$ : $\mathrm{S}(\mathrm{U}(1) \times \mathrm{U}(1))$ structure group 32

4.3 Split spectral covers with torsional points 33

4.3.1 Structure group $\mathbb{Z}_{2}: \mathrm{E}_{8} \times \mathrm{E}_{7} \times \mathrm{SU}(2)$ gauge symmetry 33

4.3.2 Structure group $\mathrm{S}\left(\mathrm{U}(2) \times \mathbb{Z}_{2}\right): \mathrm{E}_{8} \times \mathrm{E}_{6} \times \mathrm{U}(1)$ gauge symmetry 35

$4.4 \mathrm{U}(1)$ factors arising from purely non-Abelian structure groups 37

5 Conclusions and future directions $\quad 38$

A Weierstrass and Tate form of the hypersurface $\chi \quad 40$

A.1 The map to Weierstrass normal form 42 
B Spectral cover examples with no U(1) 42

B.1 Trivial structure group: $\mathrm{E}_{8} \times \mathrm{E}_{8}$ gauge symmetry 42

B.2 Structure group $\mathrm{SU}(1) \times \mathrm{SU}(2): \mathrm{E}_{8} \times \mathrm{E}_{7}$ gauge symmetry 43

B.3 Example with gauge group $\mathrm{E}_{8} \times \mathrm{SO}(11)$

C Tuned models without rational sections 45

D Non-commutativity of the semi-stable degeneration limit and the map to Weierstrass form $\quad 45$

\section{Introduction and summary of results}

The study of effective theories of string theory in lower dimensions with minimal supersymmetry are both of conceptual and phenomenological relevance. Two very prominent avenues to their construction are Calabi-Yau compactifications of the $\mathrm{E}_{8} \times \mathrm{E}_{8}$ heterotic string and of F-theory, respectively. The defining data of the two compactifications are seemingly very different. While a compactification to $10-2 n$ dimensions is defined in the heterotic string by a complex $n$-dimensional Calabi-Yau manifold $Z_{n}$ and a holomorphic, semi-stable vector bundle $V[1,2]$, in F-theory one needs to specify a complex $(n+1)$ dimensional elliptically-fibered Calabi-Yau manifold $X_{n+1}$ [3-5]. For an elliptic K3-fibered $X_{n+1}$ and an elliptically fibered $Z_{n}$, however, both formulations of compactifications of string theory are physically equivalent. The defining data of both sides are related to each other by heterotic/F-theory duality [3-5]. Most notably, this duality allows making statements about the heterotic vector bundle $V$ in terms of the controllable geometry of the Calabi-Yau manifold $X_{n+1}$ on the F-theory side. Studying the structure of the heterotic vector bundle $V$ is crucial for understanding the gauge theory sector of the resulting effective theories. In this note, we present key steps towards developing the geometrical duality map between heterotic and F-theory compactifications with Abelian gauge symmetries in their effective theories.

Since the advent of F-theory, the matching of gauge symmetry and the matter content in the effective theories has been studied in heterotic/F-theory duality [3-5]. Mathematically, the duality astonishingly allows to use the data of singular Calabi-Yau manifolds $X_{n+1}$ in F-theory to efficiently construct vector bundles $V$ on the heterotic side, which is typically very challenging. The duality can be precisely formulated in the so-called stable degeneration limit of $X_{n+1}$ [6], in which its K3-fibration degenerates into two half K3-fibrations $X_{n+1}^{ \pm}$,

$$
X_{n+1} \rightarrow X_{n+1}^{+} \cup Z_{n} X_{n+1}^{-},
$$

that intersect in the heterotic Calabi-Yau manifold, $X_{n+1}^{+} \cap X_{n+1}^{-}=Z_{n}$. It can be shown that $X_{n+1}^{ \pm}$naturally encode the heterotic vector bundle $V$ on elliptically fibered Calabi-Yau manifolds $Z_{n}$ [7]. The most concrete map between the data of $X_{n+1}$ in stable degeneration 
and the heterotic side is realized if $V$ is described by a spectral cover employing the FourierMukai transform [7, 8] (for more details see e.g. [9] and references therein). Heterotic/Ftheory duality has been systematically applied using toric geometry for the construction of vector bundles $V$ with non-Abelian structure groups described both via spectral covers and half K3 fibrations, see e.g. [10, 11] for representative works. More recently, heterotic/Ftheory duality has been used to study the geometric constraints on both sides of the duality in four-dimensional compactifications and to characterize the arising low-energy physics [12], see also [13]. Furthermore, computations of both vector bundle and M5brane superpotentials could be performed by calculation of the F-theory superpotential using powerful techniques from mirror symmetry [14-16]. In addition, the heterotic/Ftheory duality has been recently explored for studies of moduli-dependent prefactor of M5-instanton corrections to the superpotential in F-theory compactifications $[17,18]$. The focus of all these works has been on vector bundles $V$ with non-Abelian structure groups, see however $[19,20]$ for first works on aspects of heterotic/F-theory duality with U(1)'s.

In this work, we will apply the simple and unifying description on the F-theory side in terms of elliptically fibered Calabi-Yau manifolds $X_{n+1}$ to study explicitly, using stable degeneration, the structure of spectral covers yielding heterotic vector bundles that give rise to $\mathrm{U}(1)$ gauge symmetry in the lower-dimensional effective theory, continuing the analysis explained in the 2010 talk [21]. ${ }^{1}$

Abelian gauge symmetries are desired ingredients for controlling the phenomenology both of extensions of the standard model as well as of GUT theories. Recently, there has been tremendous progress on the construction of F-theory compactifications with Abelian gauge symmetries based on the improved understanding of elliptically fibered Calabi-Yau manifold $X_{n+1}$ with higher rank Mordell-Weil group of rational sections, see the representative works [22-31]. In contrast, it has been long known that Abelian gauge symmetries in the heterotic theory can for example be constructed by considering a background bundle $V$ with line bundle components [1]. The setup we are studying in this work is the duality map between the concrete and known geometry of the Calabi-Yau manifold $X_{n+1}$ with a rank one Mordell-Weil group in [22] on the F-theory side and the data of the Calabi-Yau manifold $Z_{n}$ and the vector bundle $V$ defining the dual heterotic compactification. We will demonstrate, at the hand of a number of concrete examples, the utility of the F-theory Calabi-Yau manifold $X_{n+1}$ for the construction of vector bundles with non-simply connected structure groups that arise naturally in this duality. In particular, the F-theory side will guide us to the physical interpretation of less familiar or novel structures in the heterotic vector bundle.

There are numerous key advancements in this direction presented in this work:

- We rigorously perform the stable degeneration limit of a class of F-theory Calabi-Yau manifolds $X_{n+1}$ with U(1) Abelian gauge symmetry using toric geometry, applying and extending the techniques of [32]. We explicitly extract the data of the two half-K3 surfaces inside $X_{n+1}^{ \pm}$, the spectral covers and the heterotic Calabi-Yau manifold $Z_{n}$. We point out the non-commutativity of the stable degeneration limit and birational

\footnotetext{
${ }^{1}$ We have recently learned that A. Braun and S. Schäfer-Nameki have been working on similar techniques.
} 
maps, such as the one to the Weierstrass model. The stable degeneration limit we perform, which we denote as "toric stable degeneration", preserves the structure of the Mordell-Weil group of rational sections before and after the limit, which is, in contrast, obscured in the stable degeneration limit performed in the Weierstrass model. We apply our general techniques to Calabi-Yau manifolds with elliptic fiber in $\mathrm{Bl}_{1} \mathbb{P}^{2}(1,1,2)$, which yield one $\mathrm{U}(1)$ in F-theory [22].

- We illuminate the systematics in the mapping under heterotic/F-theory duality between F-theory with a Mordell-Weil group and heterotic vector bundles with nonsimply connected structure groups leading to U(1)'s in their effective theories. We find that a single type of F-theory geometry $X_{n+1}$ can be dual to a whole range of different phenomena in the heterotic string, at the hand of numerous concrete examples. We find three different classes of examples of how a U(1) gauge group is obtained in the heterotic string: one class of examples has a split spectral cover, which is a well-known ingredient for obtaining $\mathrm{U}(1)$ gauge groups in the heterotic literature starting with [33] and the F-theory literature, see e.g. [34-36]; another class of models have a spectral cover containing a torsional section of the heterotic Calabi-Yau manifold $Z_{n}$, where duality suggests that this should describe zero-size instantons of discrete holonomy, as considered in [37]; in a last set of examples, the $\mathrm{U}(1)$ arises as the commutant inside $\mathrm{E}_{8}$ of vector bundles with purely non-Abelian structure groups. We analyze the emerging spectral covers by explicit computations in the group law on the elliptic curve in $Z_{n}$. In the first two classes of examples, it is crucial that the heterotic elliptic fibration $Z_{n}$ exhibits rational sections, as also found in [38]. In addition, in certain examples, the $\mathrm{U}(1)$ is only visible in the half $\mathrm{K} 3$ fibration (and in $Z_{n}$ ), but not in the spectral cover.

- Whereas the number of massless U(1)'s on the F-theory side equals the Mordell-Weil rank of $X_{n+1}$, it is on the heterotic side a mixture of geometry and effective field theory effects: while the analysis of the spectral cover can be performed already in $8 \mathrm{D}$, in $6 \mathrm{D}$ and lower dimensions $\mathrm{U}(1)$ 's can be lifted from the massless spectrum by a Stückelberg effect, i.e. gaugings of axions [1]. We understand explicitly in all three classes of examples how these gaugings arise and what is the remaining number of massless U(1) fields.

We note that although our analysis is performed in $8 \mathrm{D}$ and $6 \mathrm{D}$, it is equally applicable also to heterotic/F-theory duality for compactifications to $4 \mathrm{D}$.

This paper is organized in the following way: in section 2, we provide a brief review of the key points of heterotic/F-theory duality as well as a discussion of the new insights gained in this work into spectral covers and half K3-fibrations for vector bundles with non-simply connected structure groups. We review and discuss heterotic/F-theory duality in $8 \mathrm{D}$ and $6 \mathrm{D}$, the spectral cover construction for $\mathrm{SU}(N)$ bundles, specializations thereof giving rise to $\mathrm{U}(1)$ factors in the heterotic string and the Stückelberg mechanism rendering certain $\mathrm{U}(1)$ gauge fields massive. Section 3 contains the toric description of a class of F-theory models $X_{n+1}$ for which we describe a toric stable degeneration limit. We specialize to the toric 
fiber $\mathrm{Bl}_{1} \mathbb{P}^{(1,1,2)}$ and obtain the half K3-fibrations as well as the dual heterotic geometry and spectral cover polynomial. In section 4 , we present selected examples of F-theory/heterotic dual compactifications. We illustrate the three different classes of examples with heterotic vector bundles of structure groups $\mathrm{S}(\mathrm{U}(n) \times \mathrm{U}(1))$ and $\mathrm{S}\left(\mathrm{U}(n) \times \mathbb{Z}_{k}\right)$, as well as purely non-Abelian ones having a centralizer in $\mathrm{E}_{8}$ with one $\mathrm{U}(1)$ factor. There we also illustrate the utility of the Stückelberg mechanism to correctly match the number of geometrically massless U(1)'s on both sides of the duality. In section 5, we conclude and discuss possibilities for future works. This work has four appendices: we present the birational map of the quartic in $\mathbb{P}^{(1,1,2)}$ to Tate and Weierstrass form in appendix A; appendix B contains examples with no $\mathrm{U}(1)$ factor, consistently reproducing [4]; in appendix $\mathrm{C}$ we state the condition for the existence of two independent rational sections and appendix D illustrates explicitly the non-commutativity of the stable degeneration limit and the birational map to Weierstrass form.

\section{Heterotic/F-theory duality and U(1)-factors}

The aim of this section is two-fold: on the one hand, we review those aspects of heterotic/Ftheory duality in $8 \mathrm{D}$ and $6 \mathrm{D}$ that are relevant for the analyses performed in this work. On the other hand, we point out subtleties and new insights into heterotic/F-theory duality with Abelian U(1) factors. In particular, we discuss in detail split spectral covers for heterotic vector bundles with non-simply connected gauge groups and the heterotic Stückelberg mechanism.

In section 2.1, we discuss the fundamental duality in $8 \mathrm{D}$, the standard stable degeneration limit in Weierstrass form and the principal matching of gauge groups and moduli. There, we also discuss a subtlety in performing the stable degeneration limit of F-theory models with $\mathrm{U}(1)$ factors due to the non-commutativity of this limit with the map to the Weierstrass model. Section 2.2 contains a discussion of the spectral cover construction for $\mathrm{SU}(N)$ bundles as well as of split spectral covers giving rise to $\mathrm{S}(\mathrm{U}(N-1) \times \mathrm{U}(1))$ and $\mathrm{S}\left(\mathrm{U}(N-1) \times \mathbb{Z}_{k}\right)$ bundles. In section 2.3 we briefly review heterotic/F-theory duality in $6 \mathrm{D}$, before we discuss the Stückelberg effect in the effective theory of heterotic compactifications with $\mathrm{U}(1)$ bundles as well as the relation to gluing condition of rational sections in section 2.4.

In the review part, we mainly follow $[7,9,39]$, to which we refer for further details.

\subsection{Heterotic/F-theory duality in $8 \mathrm{D}$}

The basic statement of heterotic/F-theory duality is that the heterotic string (in the following, we always concentrate on the $\mathrm{E}_{8} \times \mathrm{E}_{8}$ string) compactified on a torus, which we denote by $Z_{1}$, is equivalent to F-theory compactified on an elliptically fibered K3 surface $X_{2}$. The first evidence is that the moduli spaces $\mathcal{M}$ of these two theories coincide and are parametrized by

$$
\mathcal{M}=\mathrm{SO}(18,2, \mathbb{Z}) \backslash \mathrm{SO}(18,2, \mathbb{R}) /(\mathrm{SO}(18) \times \mathrm{SO}(2)) \times \mathbb{R}^{+} .
$$


From a heterotic perspective this is just the parametrization of the complex and Kähler structure of the torus $Z_{1}$ as well as of the 24 Wilson lines. On the F-theory side it corresponds to the moduli space of algebraic K3 surfaces $X_{2}$ with Picard number two. The last factor corresponds to the vacuum expectation value of the dilaton and the size of the base $\mathbb{P}^{1}$ of $X_{2}$, respectively.

Lower-dimensional dualities are obtained, applying the adiabatic argument [40], by fibering the eight-dimensional duality over a base manifold $B_{n-1}$ of complex dimension $n-1$ that is common to both theories of the duality.

\subsubsection{The standard stable degeneration limit}

In order to match the moduli on both sides of the duality, the K3 surface $X_{2}$ has to undergo the so-called stable degeneration limit. In this limit it splits into two half K3 surfaces $X_{2}^{+}$, $X_{2}^{-}$as

$$
X_{2} \rightarrow X_{2}^{+} \cup_{Z_{1}} X_{2}^{-}
$$

Each of these are an elliptic fibration $\pi_{ \pm}: X_{2}^{ \pm} \longrightarrow \mathbb{P}^{1}$ over a $\mathbb{P}^{1}$. These two $\mathbb{P}^{1}$ 's intersect in precisely one point so that the two half $\mathrm{K} 3$ surfaces intersect in a common elliptic fiber which is identified with the heterotic elliptic curve, $X_{2}^{+} \cap X_{2}^{-}=Z_{1}$. On the heterotic side, the stable degeneration limit corresponds to the large elliptic fiber limit of $Z_{1}$.

Matching the gauge groups. The F-theory gauge group is given by the singularities of the elliptic fibration of $X_{2}$, determining the non-Abelian part $G$, and its rational sections, which correspond to Abelian gauge fields [3, 5, 41]. In stable degeneration the non-Abelian gauge group of F-theory is distributed into the two half K3 surfaces $X_{2}^{ \pm}$and matched with the heterotic side as follows.

It is a well-known fact that the homology lattice of a half $\mathrm{K} 3$ surface $X_{2}^{ \pm}$is given in general by

$$
H_{2}\left(X_{2}^{ \pm}, \mathbb{Z}\right)=\Gamma_{8} \oplus U
$$

Here, $U$ contains the classes of the elliptic fiber as well as of the zero section. $\Gamma_{8}$ equals the root lattice of $\mathrm{E}_{8}$ and splits into a direct sum of two contributions [42]: the first contribution is given by the Mordell-Weil group of the rational elliptic surface while the second contribution is given by a sub-lattice which forms, for the half K3 surfaces $X_{2}^{ \pm}$at hand, the root-lattice of the part $G_{ \pm}$of the non-Abelian F-theory gauge group $G=G_{+} \times G_{-}$ that is of ADE type. In the F-theory limit all fiber components are shrunken to zero size and the half $\mathrm{K} 3$ surface develops a singularity of type $G_{ \pm}$. The possible ADE-singularities in the case of complex surfaces have been classified by Kodaira [43]. Thus, one can always read off the corresponding gauge group from the order of vanishings of $f, g$ and $\Delta$ once the half K3 has been brought into affine Weierstrass normal form

$$
y^{2}=x^{3}+f x z^{4}+g z^{6}, \quad \Delta=4 f^{3}+27 g^{2},
$$

with $f$ and $g$ in $\mathcal{O}(4)$ and $\mathcal{O}(6)$ of $\mathbb{P}^{1}$, respectively. For convenience of the reader, we reproduce Kodaira's classification in table 1. 


\begin{tabular}{|c|c|c|c|}
\hline order $(f)$ & order $(g)$ & order $(\Delta)$ & singularity \\
\hline$\geqslant 0$ & $\geqslant 0$ & 0 & none \\
0 & 0 & $n$ & $\mathrm{~A}_{n-1}$ \\
$\geqslant 1$ & 1 & 2 & none \\
1 & $\geqslant 2$ & 3 & $\mathrm{~A}_{1}$ \\
$\geqslant 2$ & 2 & 4 & $\mathrm{~A}_{2}$ \\
2 & $\geqslant 3$ & $n+6$ & $\mathrm{D}_{n+4}$ \\
$\geqslant 2$ & 3 & $n+6$ & $\mathrm{D}_{n+4}$ \\
$\geqslant 3$ & 4 & 8 & $\mathrm{E}_{6}$ \\
3 & $\geqslant 5$ & 9 & $\mathrm{E}_{7}$ \\
$\geqslant 4$ & 5 & 10 & $\mathrm{E}_{8}$ \\
\hline
\end{tabular}

Table 1. The Kodaira classification of singular fibers. Here $f$ and $g$ are the coefficients of the Weierstrass normal form, $\Delta$ is the discriminant as defined in (2.4) and order refers to their order of vanishing at a particular zero.

In contrast, the gauge group on the heterotic side is encoded in two vector bundles $V_{1}$, $V_{2}$ that generically carry the structure group $\mathrm{E}_{8}$. Their respective commutants inside the two ten-dimensional $\mathrm{E}_{8}$ gauge groups of the heterotic string are to be identified with the F-theory gauge group. As observed in [7], the moduli space of semi-stable $\mathrm{E}_{8}$-bundles on an elliptic curve $E$ corresponds to the complex structure moduli space of a half K3 surface $S$ whose anti-canonical class is given by $E$. Furthermore, if $S$ has an ADE singularity of type $\tilde{G}_{ \pm}$then the structure group of $V_{1}, V_{2}$ is reduced to the centralizer $H_{ \pm}$of $\tilde{G}_{ \pm}$within $\mathrm{E}_{8}$, respectively. In heterotic/F-theory duality, a matching of the gauge group is then established by identifying $S \equiv X_{2}^{ \pm}$yielding $\tilde{G}_{ \pm} \equiv G_{ \pm}$.

Notice that the full eight-dimensional gauge group is given by $G \times \mathrm{U}(1)^{16-\mathrm{rk}(G)} \times \mathrm{U}(1)^{4}$. Here, the last factor accounts for the reduction of the metric and the Kalb Ramond $B$-field along the two one-cycles of the torus in the heterotic string. From the F-theory perspective, all U(1) factors arise from the reduction of the $C_{3}$ field along those 2-forms in the full K3 surface $X_{2}$ that are orthogonal to the zero section and the elliptic fiber. In particular, the $\mathrm{U}(1)^{16-\mathrm{rk}(G)}$ arises from the generators of the Mordell-Weil group of the half K3 surfaces. For a derivation in Type IIB string theory, see the recent work [44].

Matching complex structure and bundle moduli. In this section, we discuss how the heterotic moduli can be recovered from the data of the F-theory K3 surface [4, 45]. Here we restrict the discussion to the moduli of the heterotic torus $Z_{1}$ and the vector bundle (i.e. Wilson line) moduli, ignoring the heterotic dilaton modulus.

So far, this discussion has been restricted to the case that the elliptic fibration of the K3 surface is described by a Weierstrass model. In this case, the standard stable degeneration procedure applies. Given the Weierstrass form (2.4) for $X_{2}$ with $f, g$ sections of $\mathcal{O}(8)$ and $\mathcal{O}(12)$ on $\mathbb{P}^{1}$, respectively, we can expand these degree eight and twelve polynomials in the affine $\mathbb{P}^{1}$-coordinate $u$ as

$$
f=\sum_{i=0}^{8} f_{i} u^{i}, \quad g=\sum_{i=0}^{12} g_{i} u^{i} .
$$


Then, the two half K3 surfaces $X_{2}^{ \pm}$arising in the stable degeneration limit, given as the Weierstrass models

$$
X_{2}^{ \pm}: \quad y^{2}=x^{3}+f^{ \pm} z^{4}+g^{ \pm} z^{6},
$$

can be obtained from (2.5) by the split

$$
f^{+}=\sum_{i=0}^{4} f_{i} u^{i}, \quad f^{-}=\sum_{i=4}^{8} f_{i} u^{i}, \quad g^{+}=\sum_{i=0}^{6} g_{i} u^{i}, \quad g^{-}=\sum_{i=6}^{12} g_{i} u^{i},
$$

The "middle" polynomials $f_{4}$ and $g_{6}$ correspond to the heterotic elliptic curve, which then reads

$$
Z_{1}: \quad y^{2}=x^{3}+f_{4} x z^{4}+g_{6} z^{6},
$$

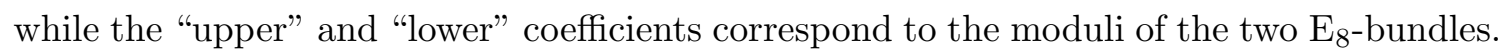

\subsubsection{Stable degeneration with other elliptic fiber types}

The focus of the present work are F-theory compactifications with one $\mathrm{U}(1)$ gauge group arising from elliptically fibered Calabi-Yau manifolds with two rational sections. These are naturally constructed using the fiber ambient space $\mathrm{Bl}_{1} \mathbb{P}^{(1,1,2)}[22]$. More precisely, we will consider K3 surfaces given as sections $\chi$ of the anti-canonical bundle $-K_{\mathbb{P}^{1} \times \mathrm{Bl}_{1} \mathbb{P}^{(1,1,2)}}$ of $\mathbb{P}^{1} \times \mathrm{Bl}_{1} \mathbb{P}^{(1,1,2)}$ reading

$$
\chi=\sum_{i} s_{i} \chi^{i}
$$

Here $s_{i}$ and $\chi^{i}$ are sections of the anti-canonical bundles $-K_{\mathbb{P}^{1}}=\mathcal{O}(2)$ and $-K_{\mathrm{Bl}_{1} \mathbb{P}^{(1,1,2)}}$, respectively.

Then, analogously to the above construction, one can perform a stable degeneration limit for these hypersurfaces as well. However, it is crucial to note here that we can perform the stable degeneration limit in two possible ways, as shown in figure 1: one way is to first take the Weierstrass normal form $W_{\chi}$ (upper horizontal arrow) of the full $\mathrm{Bl}_{1} \mathbb{P}^{(1,1,2)}$-model and then apply the split (2.7) to obtain two half K3 surfaces (right vertical arrow); a second way is to first perform stable degeneration (left vertical arrow), yielding two half K3 surfaces $\chi^{ \pm}$with elliptic fibers in $\mathrm{Bl}_{1} \mathbb{P}^{(1,1,2)}$, and then compute their Weierstrass normal forms $W_{\chi}^{ \pm}$ (lower horizontal arrow). It is important to realize, however, that these two possible paths in the diagram 1 do not commute, as explicitly shown in appendix D.

We propose and demonstrate in section 3 that the natural order to perform heterotic/Ftheory duality for models with $\mathrm{U}(1)$ factors and different elliptic fiber types than the Weierstrass model is to first perform stable degeneration with the other fiber type (left vertical arrow) and then compute the Weierstrass model of the resulting half K3-fibrations (lower horizontal arrow) in order to analyze the physics of the model.

\subsection{Constructing $\mathrm{SU}(N)$ bundles on elliptic curves and fibrations}

While the description of the structure group of the vector bundle via half K3 surfaces as reviewed above is of high conceptual importance, it is in practice often easier to construct vector bundles with the desired structure group directly. In the following section, we review 


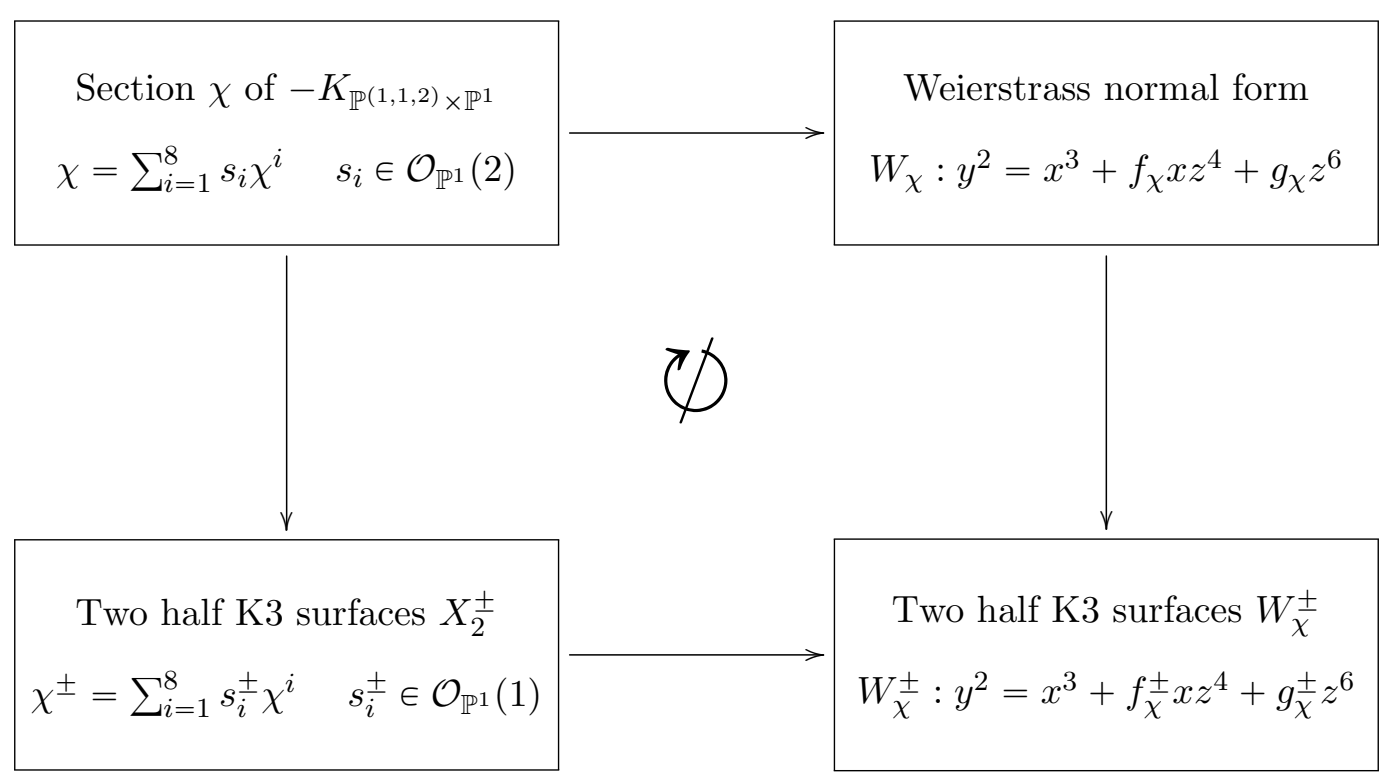

Figure 1. Computing the Weierstrass normal form (horizontal arrows) and taking the stable degeneration limit (vertical arrows) does not commute.

this construction for $\mathrm{SU}(N)$ bundles and specializations thereof which has been studied first in [46] and was further developed in [7, 8, 47].

In this section $E$ always denotes an elliptic curve with a marked point $p$. The curve is defined over a general field $K$, which does not necessarily have to be algebraically closed. It is well-known that an elliptic curve with a point $p$ has a representation in the Weierstrass normal form $(2.4)$, where $p$ reads $[x: y: z]=[1: 1: 0]$. In general, a degree zero line bundle $\mathcal{L} \longrightarrow E$, i.e. a $\mathrm{U}(1)$-bundle, takes the form

$$
\mathcal{L}=\mathcal{O}(q) \otimes \mathcal{O}(p)^{-1}=\mathcal{O}(q-p)
$$

where $q$ denotes another arbitrary rational point on $E$ (note that over $K=\mathbb{C}$ every point is rational). Furthermore, we note that there is a bijective map $\phi$ from the elliptic curve $E$ onto its Picard group of degree zero which is defined by

$$
\phi: E \longrightarrow \operatorname{Pic}^{0}(E), \quad q \mapsto q-p
$$

In particular, this extends to an isomorphism from the space of line bundles onto $\operatorname{Pic}^{0}(E)$, defined by $\operatorname{div}(\mathcal{L})=q-p$. To be more precise, the divisor map 'div' is to be applied to a meromorphic section ${ }^{2}$ of $\mathcal{L}$. For later purposes, we also recall that the addition law in $\operatorname{Pic}^{0}(E)$ can be identified with the group law on $E$, which we denote by $\boxplus$, via this isomorphism.

\footnotetext{
${ }^{2}$ This map is independent of the section chosen.
} 
A semi-stable $\mathrm{SU}(N)$ vector bundle of degree zero $V$ is then given as the $\operatorname{sum}^{3}$ of $N$ holomorphic line bundles $\mathcal{L}_{i}$, i.e. we have $V=\oplus_{i=1}^{N} \mathcal{L}_{i}=\oplus_{i=1}^{N} \mathcal{O}\left(q_{i}-p\right)$, such that the determinant of $V$ is trivial. The latter implies that

$$
\otimes_{i=1}^{N} \mathcal{O}\left(q_{i}-p\right)=\mathcal{O} \quad \Leftrightarrow \quad \bigoplus_{i=1}^{N} q_{i}=0 .
$$

An $\mathrm{SU}(N)$ vector bundle is therefore determined by the choice of $N$ points on $E$ that sum up to zero. Any such $N$-tupel is determined by a projectively unique element of $H^{0}(E, \mathcal{O}(N p))$, i.e. a function with $N$ zeros and a pole of order $N$ at $p$. Thus, the moduli space of $\mathrm{SU}(N)$ vector bundles is given by

$$
\mathcal{M}_{\mathrm{SU}(N)}=\mathbb{P} H^{0}(E, \mathcal{O}(N p)) .
$$

In the affine Weierstrass form of $E$, given by (2.4) with $z$ set to one, the coordinates $x, y$ have a pole of order two and three at $p$, respectively. Accordingly, any element of $\mathbb{P} H^{0}(E, \mathcal{O}(N p))$ enjoys an expansion

$$
w=c_{0}+c_{1} x+c_{2} y+c_{3} x^{2}+\ldots+ \begin{cases}c_{N} x^{\frac{N}{2}} & \text { if } N \text { is even } \\ c_{N} x^{\frac{N-3}{2}} y & \text { if } N \text { is odd }\end{cases}
$$

with $c_{i} \in K$. The section $w$ is called the spectral cover polynomial and has $N$ common points with $E$, called the spectral cover, which define the desired $\mathrm{SU}(N)$ bundle. Counting parameters of (2.14), one is lead to the conclusion that

$$
\mathcal{M}_{\mathrm{SU}(N)}=\mathbb{P}^{N-1} \text {. }
$$

Finally, a comment on rational versus non-rational points is in order. Generically, $p$ is the only point on $E$ over a general field $K$. However, in such a situation, it is possible to mark $N$ points in a rational way by the polynomial $w=0$ which give rise to an $S U(N)$ bundle in the way just described. Nevertheless, under the circumstances that there are additional rational points on $E$ and the spectral cover polynomial $w=0$ specializes appropriately, the structure group reduces in a certain way, as discussed next.

\subsubsection{Vector bundles with reduced structure groups}

As described in the previous section, the choice of $N$ points on $E$ describes an $\mathrm{SU}(N)$ bundle. If we consider just an elliptic curve $E$ over $\mathbb{C}$, which is the geometry relevant for the construction of heterotic compactifications to $8 \mathrm{D}$, the spectral cover (2.14) can be factorized completely. This corresponds to the 16 possible Wilson lines on $T^{2}$.

In contrast, if we consider an elliptic curve over a function field, as it arises in elliptic fibrations $Z_{n}$ of $E$ over a base $B_{n-1}$ used for lower-dimensional heterotic compactifications, the $N$ points are the zeros of (2.14), which defines an $N$-section of the fibration. In nongeneric situations, where subsets of the $N$ sheets of this $N$-section are well-defined globally,

\footnotetext{
${ }^{3}$ If two or more points coincide, the situation is a bit more subtle [47, 48]. In this case the bundle is given by $\oplus_{i=1}^{N} \mathcal{O}\left(q_{i}-p\right) I_{r_{i}}$, where $r_{i}$ denotes the multiplicity of the point $q_{i}$ and $I_{r}$ is inductively defined by the extension sequence $0 \longrightarrow \mathcal{O} \longrightarrow I_{r-1} \longrightarrow \mathcal{O} \longrightarrow 0$. However, one usually only considers bundles up to S-equivalence which identifies $I_{r}$ with $\mathcal{O}^{\oplus r}$.
} 
i.e. are monodromy invariant, the structure group of the vector bundle is reduced. For example, a separation into two sets of $k$ and $l$ sheets (with $k+l=N$ ), respectively, results in the structure group $\mathrm{S}(\mathrm{U}(k) \times \mathrm{U}(l))$. The spectral cover defined by $(2.14)$ is called "split" and defines a reducible variety inside $Z_{n}$, see e.g. [33-36]. In the most extreme case, one could have $k=1$ and $l=N-1$. In this case, the elliptic fibration of $Z_{n}$ has to necessarily have another well-defined section in addition to the section induced by the rational point $p$ : it is the one marked by the component of the spectral cover $w=0$ with just one sheet [38]. Thus, the fiber $E$ has a rational point, which we denote by $q$ and one can, as discussed above, define a $\mathrm{U}(1)$ line bundle $\mathcal{L}$ via (2.10). As this fiberwise well-defined line bundle is also well-defined globally, it will induce a line bundle on $Z_{n}$, whose first Chern class is given, up to vertical components, by the difference of the sections induced by $q$ and $p$, cf. [49]. The structure group $H$ of the vector bundle is in this case given by

$$
H=\mathrm{S}(\mathrm{U}(N-1) \times \mathrm{U}(1))
$$

We will see later that this situation will be relevant situation for the construction of $\mathrm{U}(1)$ gauge groups in the heterotic string.

We emphasize that for a $\mathrm{U}(1)$-bundle alone there is no spectral cover polynomial (2.14) that would be able to detect this additional rational point. This is due to the fact that there is no function that has only one zero on an elliptic curve $E$. However, if the rational point is accompanied by further points, rational or non-rational points over the field $K$, it can very well be seen by the spectral cover. For instance, one could construct a spectral cover from $q$ and $-q$, which would describe a bundle of structure group $\mathrm{S}(\mathrm{U}(1) \times \mathrm{U}(1))$.

Finally, it needs to be discussed what interpretation should be given to the case that the rational point $q$ on the curve $E$ happens to be torsion of order $k$. In this case the structure group $H$ reduces further to $\mathrm{S}\left(\mathrm{U}(N) \times \mathbb{Z}_{k}\right)$. To argue for this, we invoke again a fiberwise argument. The fiber at a generic point in $B_{n-1}$ admits a line bundle $\mathcal{L}=\mathcal{O}(q-p)$ with the property that $\mathcal{L}^{k}=\mathcal{O}$. This is clear as the transition functions $g_{i j}$ will be subject to $g_{i j}^{k}=1$ in Čech cohomology as $k$ times the Poincaré dual of its first Chern class is trivial. However, this is just the statement that the fiberwise structure group of $\mathcal{L}$ is contained in $\mathbb{Z}_{k}$. Employing that $p$ and $q$ are globally well-defined sections then suggests that this argument also holds on $Z_{n}$.

\subsection{Heterotic/F-theory duality in 6D}

Six-dimensional heterotic/F-theory duality arises by fibering the eight-dimensional duality over a common base $B_{1}=\mathbb{P}^{1}$, employing the adiabatic argument [40]. Thus, the heterotic string gets compactified on an elliptically fibered $\mathrm{K} 3$ surface $Z_{2}$ while F-theory is compactified on an elliptically fibered Calabi-Yau threefold $X_{3}$ over a Hirzebruch surface $\mathbb{F}_{n}$. Our presentation will be brief and focused on the later applications in this work. For a more detailed discussion we refer to the classical reference $[4,5,7]$ or the reviews $[9,39]$.

On the F-theory side, the non-Abelian gauge content originates from the codimension one singularities of the elliptic fibration $\pi: X_{3} \rightarrow \mathbb{F}_{n}$. The singularity is generically of type $G^{\prime}$, which gets broken down to $G \subset G^{\prime}$ by monodromies corresponding to outer 
automorphisms of the Dynkin diagram of $G^{\prime}$ [41]. The resulting gauge symmetry is encoded in the order of vanishing of the coefficients $a_{0}, a_{1}, a_{2}, a_{3}, a_{4}, a_{6}$ in the Tate form of the elliptic fibration

$$
y^{2}+a_{1} x y+a_{2}=x^{3}+a_{3} x^{2}+a_{4} x+a_{6} .
$$

In addition, we introduce the Tate vector $\vec{t}_{X}$ which encodes the orders of vanishing of the coefficients $a_{i}$ along the divisor defined by the local coordinate $X$ :

$$
\vec{t}_{X}=\left(\operatorname{ord}_{X}\left(a_{0}\right), \operatorname{ord}_{X}\left(a_{1}\right), \operatorname{ord}_{X}\left(a_{2}\right), \operatorname{ord}_{X}\left(a_{3}\right), \operatorname{ord}_{X}\left(a_{4}\right), \operatorname{ord}_{X}\left(a_{6}\right), \operatorname{ord}_{X}(\Delta)\right) .
$$

The results of the analysis of singularities, known as Tate's algorithm, are summarized in table $2[41,50]$, see, however, [51] for subtleties.

On the heterotic side, the gauge theory content is encoded in a vector bundle $V$ where the following discussion restricts itself to the case of $\mathrm{SU}(N)$ bundles. The six-dimensional bundle is defined in terms of two pieces of data, the spectral cover curve $C$ as well as a line bundle $\mathcal{N}$ which is defined on $C$. Here, the spectral curve $C$ is the $6 \mathrm{D}$ analog of the points defined by the section of $\mathbb{P} H^{0}(E, \mathcal{O}(n p))$ which has been discussed in 2.2 . In $6 \mathrm{D}$, the elliptic curve $Z_{1} \cong E$ gets promoted to an elliptic fibration, which can again be described by a Weierstrass form (2.4) with coordinates $x, y, z$ being sections of $\mathcal{L}^{2}, \mathcal{L}^{2}, \mathcal{O}$, respectively, for $\mathcal{L}=K_{\mathbb{P}^{1}}^{-1}=\mathcal{O}(-2)$ and coefficients $f, g$ being in $\mathcal{L}^{4}, \mathcal{L}^{6}$, respectively. Accordingly, the coefficients $c_{i}$ entering the spectral cover (2.14) are now sections of $\mathcal{M} \otimes \mathcal{L}^{-i}, \mathcal{M}$ being an arbitrary line bundle on $\mathbb{P}^{1}$ and $C$ is defined as the zero locus of the section of (2.14). Thus, $C$ defines an $N$-sheeted ramified covering of $\mathbb{P}^{1}$, i.e. a Riemann surface. The spectral cover $C$ defines the isomorphism class of a semi-stable vector bundle above each fiber. The line bundle $\mathcal{N}$ describes the possibility to twist the vector bundle without changing its isomorphism class. It is usually fixed, up to a twisting class $\gamma$, by the condition $c_{1}(V)=0$ for an $\mathrm{SU}(N)$ bundle, see [7] for more details.

\subsection{Massless U(1)-factors in heterotic/F-theory duality}

As previously discussed, the perturbative heterotic gauge group is obtained by commuting the structure group $H$ of the vector bundle $V$ within the two $\mathrm{E}_{8}$-bundles. We propose three possibilities, how $\mathrm{U}(1)$ gauge groups can arise from this perspective:

- $H$ contains a $\mathrm{U}(1)$ factor, i.e. it is of the form $H=H_{1} \times \mathrm{U}(1)$, or $\mathrm{S}(\mathrm{U}(M) \times \mathrm{U}(1))$,

- $H$ contains a discrete piece, i.e. a part taking values in $\mathbb{Z}_{k}$,

- or $H$ is non-Abelian and is embedded such that its centralizer in $\mathrm{E}_{8}$ necessarily contains a U(1)-symmetry.

The construction of a vector bundle for these three different cases employing spectral covers has been discussed in section 2.2 .

In general, we emphasize that $\mathrm{U}(1)$-factor which arises from a split spectral cover is usually massive due to a Stückelberg mass term which is induced by the first Chern class of the U(1) background bundle, as we review next. However, if the U(1) term originates from a 


\begin{tabular}{|c|c|c|c|c|c|c|c|}
\hline Type & Group & $a_{1}$ & $a_{2}$ & $a_{3}$ & $a_{4}$ & $a_{6}$ & $\Delta$ \\
\hline $\mathrm{I}_{0}$ & $\{e\}$ & 0 & 0 & 0 & 0 & 0 & 0 \\
\hline $\mathrm{I}_{1}$ & $\{e\}$ & 0 & 0 & 1 & 1 & 1 & 1 \\
\hline $\mathrm{I}_{2}$ & $\mathrm{SU}(2)$ & 0 & 0 & 1 & 1 & 2 & 2 \\
\hline $\mathrm{I}_{3}$ & $\mathrm{SU}(3)$ & 0 & 1 & 1 & 2 & 3 & 3 \\
\hline $\mathrm{I}_{2 k}, k \geqslant 2$ & $\mathrm{Sp}(k)$ & 0 & 0 & $k$ & $k$ & $2 k$ & $2 k$ \\
\hline $\mathrm{I}_{2 k+1}, k \geqslant 1$ & $\mathrm{Sp}(k)$ & 0 & 0 & $k+1$ & $k+1$ & $2 k+1$ & $2 k+1$ \\
\hline $\mathrm{I}_{n}, n \geqslant 4$ & $\mathrm{SU}(n)$ & 0 & 1 & {$\left[\frac{n}{2}\right]$} & {$\left[\frac{n+1}{2}\right]$} & $n$ & $n$ \\
\hline II & $\{e\}$ & 1 & 1 & 1 & 1 & 1 & 2 \\
\hline III & $\mathrm{SU}(2)$ & 1 & 1 & 1 & 1 & 2 & 3 \\
\hline IV & $\mathrm{Sp}(1)$ & 1 & 1 & 1 & 2 & 2 & 4 \\
\hline IV & $\mathrm{SU}(3)$ & 1 & 1 & 1 & 2 & 3 & 4 \\
\hline $\mathrm{I}_{0}^{*}$ & $\mathrm{G}_{2}$ & 1 & 1 & 2 & 2 & 3 & 6 \\
\hline $\mathrm{I}_{0}^{*}$ & $\operatorname{Spin}(7)$ & 1 & 1 & 2 & 2 & 4 & 6 \\
\hline $\mathrm{I}_{0}^{*}$ & $\operatorname{Spin}(8)$ & 1 & 1 & 2 & 2 & 4 & 6 \\
\hline $\mathrm{I}_{1}^{*}$ & Spin(9) & 1 & 1 & 2 & 3 & 4 & 7 \\
\hline $\mathrm{I}_{1}^{*}$ & $\operatorname{Spin}(10)$ & 1 & 1 & 2 & 3 & 5 & 7 \\
\hline $\mathrm{I}_{2}^{*}$ & $\operatorname{Spin}(11)$ & 1 & 1 & 3 & 3 & 5 & 8 \\
\hline $\mathrm{I}_{2}^{*}$ & $\operatorname{Spin}(12)$ & 1 & 1 & 3 & 3 & 5 & 8 \\
\hline $\mathrm{I}_{2 k-3}^{*}, k \geqslant 3$ & $\mathrm{SO}(4 k+1)$ & 1 & 1 & $k$ & $k+1$ & $2 k$ & $2 k+3$ \\
\hline $\mathrm{I}_{2 k-3}^{*}, k \geqslant 3$ & $\mathrm{SO}(4 k+2)$ & 1 & 1 & $k$ & $k+1$ & $2 k+1$ & $2 k+3$ \\
\hline $\mathrm{I}_{2 k-2}^{*}, k \geqslant 3$ & $\mathrm{SO}(4 k+3)$ & 1 & 1 & $k+1$ & $k+1$ & $2 k+1$ & $2 k+4$ \\
\hline $\mathrm{I}_{2 k-2}^{*}, k \geqslant 3$ & $\mathrm{SO}(4 k+4)$ & 1 & 1 & $k+1$ & $k+1$ & $2 k+1$ & $2 k+4$ \\
\hline $\mathrm{IV}^{*}$ & $\mathrm{~F}_{4}$ & 1 & 2 & 2 & 3 & 4 & 8 \\
\hline $\mathrm{IV}^{*}$ & $\mathrm{E}_{6}$ & 1 & 2 & 2 & 3 & 5 & 8 \\
\hline III* & $E_{7}$ & 1 & 2 & 3 & 3 & 5 & 9 \\
\hline II* & $\mathrm{E}_{8}$ & 1 & 2 & 3 & 4 & 5 & 10 \\
\hline non-min & - & 1 & 2 & 3 & 4 & 6 & 12 \\
\hline
\end{tabular}

Table 2. Results from Tate's algorithm.

background bundle with non-Abelian structure group there is tautologically no U(1) background factor which could produce a mass term and therefore the six-dimensional U(1) field is expected to be massless. Finally, we propose, for consistency with heterotic/F-theory duality, that a six-dimensional torsional section gives rise to a point-like instanton with discrete holonomy, as introduced in [37]. Indeed, we will show in several examples in section 4 that all three cases naturally appear in heterotic duals of F-theory compactifications with one $\mathrm{U}(1)$ and that a matching of the corresponding gauge groups is only possible if the arising spectral covers are interpreted as suggested here. 


\subsubsection{The heterotic Stückelberg mechanism}

In six and lower dimensions, it is well-known that a geometric Stückelberg effect can render a U(1) gauge field massive [1]. To identify the mass term of the six- (or lower-) dimensional $\mathrm{U}(1)$, one considers the modified ten-dimensional kinetic term of the Kalb-Ramond field $B_{2}$ which reads, up to some irrelevant proportionality constant, as

$$
\mathcal{L}_{\text {kin }}^{10 d}=H \wedge \star_{10 d} H, \quad H=d B_{2}-\frac{\alpha^{\prime}}{4}\left(\omega_{3 Y}(A)-\omega_{3 L}(\Omega)\right) .
$$

Here, $\star_{10 d}$ is the ten-dimensional Hodge-star and $\omega_{3 Y}, \omega_{3 L}$ denote the Chern-Simons terms of the gauge field and the spin connection, respectively. The physical effect we want to discuss here arises from the former one, which is given explicitly by

$$
\omega_{3 Y}=\operatorname{Tr}\left(A \wedge d A+\frac{2}{3} A \wedge A \wedge A\right) .
$$

Now, we perform a dimensional reduction of the kinetic term (2.19) in the background of a $\mathrm{U}(1)$ vector bundle on the heterotic compactification manifold $Z_{n}$, ignoring possible additional non-Abelian vector bundles for simplicity. On such a background, we can expand the ten-dimensional field strength $F_{\mathrm{U}(1)}^{10 D}$ of the $\mathrm{U}(1)$ gauge field as

$$
F_{\mathrm{U}(1)}^{10 D}=F_{\mathrm{U}(1)}+\mathcal{F}=F_{\mathrm{U}(1)}+k_{\alpha} \omega^{\alpha} .
$$

Here $\mathcal{F}=\frac{1}{2 \pi i} c_{1}(\mathcal{L})$ is the background field strength, i.e. the first Chern class $c_{1}(\mathcal{L})$ of the corresponding $\mathrm{U}(1)$ line bundle $\mathcal{L}$, and $F_{\mathrm{U}(1)}$ is the lower-dimensional gauge field. We have also introduced a basis $\omega^{\alpha}, \alpha=1, \ldots, b_{2}\left(Z_{n}\right)$, of harmonic two-forms in $H^{(2)}\left(Z_{n}\right)$, where $b_{2}\left(Z_{n}\right)$ is the second Betti number of $Z_{n}$, along which we have expanded $\mathcal{F}$ into the flux quanta $k_{\alpha}$. We also expand the ten-dimensional Kalb-Ramond field as

$$
B_{2}=b_{2}+\rho_{\alpha} \omega^{\alpha},
$$

where $b_{2}$ is a lower-dimensional two-form and $\rho_{\alpha}$ are lower-dimensional axionic scalars . We readily insert this reduction ansatz into the ten-dimensional field strength $H$ in (2.19), where we only take into account the gauge part, to arrive, dropping unimportant prefactors, at the lower-dimensional kinetic term for the axions $\rho_{\alpha}$ of the form

$$
\mathcal{L}_{\text {Stück. }}=G^{\alpha \beta}\left(d \rho_{\alpha}+k_{\alpha} A_{\mathrm{U}(1)}\right) \wedge \star\left(d \rho_{\beta}+k_{\beta} A_{\mathrm{U}(1)}\right) .
$$

Here we introduced the kinetic metric

$$
G^{\alpha \beta}=\int_{Z_{n}} \omega^{\alpha} \wedge \star \omega^{\beta} .
$$

It is clear from (2.23) that a single $\mathrm{U}(1)$ gauge field will be massive if we have a nontrivial $c_{1}(\mathcal{L}) \neq 0$. However, we note that in the presence of multiple massive $\mathrm{U}(1)$ gauge fields, appropriate linear combinations of them in the kernel of the mass matrix can remain massless U(1) fields. A computation similar to the one above has appeared in e.g. [33], where also the case of multiple U(1)'s is systematically discussed. 


\subsubsection{U(1)-factors from gluing conditions in half K3-fibrations}

We conclude this section by discussing the connection between the previous field theoretic considerations that lead to a massive U(1) via the Stückelberg action (2.23) on the heterotic side and geometric glueing conditions of the sections of half K3 surfaces to global sections of the two half K3-fibrations $X_{n}^{ \pm}$that arise in stable degeneration as well as of the full Calabi-Yau manifold $X_{n}$. We illustrate this in 6D for concreteness, i.e. for F-theory on a Calabi-Yau threefold $X_{3}$ and the heterotic string on a K3 surface $Z_{2}$, although the arguments hold more generally.

It is well known that the number of $\mathrm{U}(1)$ factors in F-theory is given by the rank of the Mordell-Weil group, i.e. by the number of independent global rational sections of the elliptic fibration $X_{3}$ in addition to the zero section. As discussed in section 2.1, a half K3 surface with ADE singularity of rank $r$ has an $(8-r)$-dimensional Mordell-Weil group. Promoting the half K3 surface to a fibration of half K3 surfaces over the base $\mathbb{P}^{1}$, such as the threefolds $X_{3}^{ \pm}$, these sections need not necessarily give rise to sections of the arising three-dimensional elliptic fibrations. Considering the half K3 surfaces arising in the stable degeneration limit of F-theory, there are those sections which also give rise to sections of e.g. the full half K3 fibration $X_{3}^{+}$. These sections will induce a U(1)-factor on the heterotic side which is embedded into one $\mathrm{E}_{8}$-bundle and which is generically massive with a mass arising via the Stückelberg action (2.23). If there is also a globally well-defined section of the other half $\mathrm{K} 3$ fibration $X_{3}^{-}$and this section glues with the section in the first half K3 fibration $X_{3}^{+}$, then there is a linear combination of U(1)'s that remains massless in the Stückelberg mechanism on the heterotic side. This is clear from the F-theory perspective, as these two sections can then be glued along the heterotic two-fold $Z_{2}$ to a section of the full Calabi-Yau threefold $X_{3}$, i.e. give rise to an element in its Mordell-Weil group and a massless U(1).

\section{Dual geometries with toric stable degeneration}

In this section, we describe a toric method in order to study the stable degeneration limit of an elliptically fibered K3 surface. This stable degeneration limit will be at the heart of the analysis of the examples of heterotic/F-theory dual geometries in section 4 . In a first step in section 3.1, we construct an elliptically fibered K3 surface. Afterwards in section 3.2, we fiber this $\mathrm{K} 3$ surface over another $\mathbb{P}^{1}$ which is used to investigate the splitting of the K3 surface into two rational elliptic surfaces, as discussed in section 3.3. In the concluding section 3.4, we prove that the surfaces arising in the stable degeneration of the K3 surface indeed define rational elliptic surfaces, i.e. half K3 surfaces.

\subsection{Constructing an elliptically fibered K3 surface}

We start by constructing a three-dimensional reflexive polytope $\Delta_{3}^{\circ}$ given as the convex hull of vertices that are the rows of the following matrix:

$$
\left(\begin{array}{ccc|c}
a_{1} & b_{1} & 0 & x_{1} \\
\vdots & & 0 & x_{i} \\
a_{n} & b_{n} & 0 & x_{n} \\
0 & 0 & 1 & U \\
0 & 0 & -1 & V
\end{array}\right) .
$$



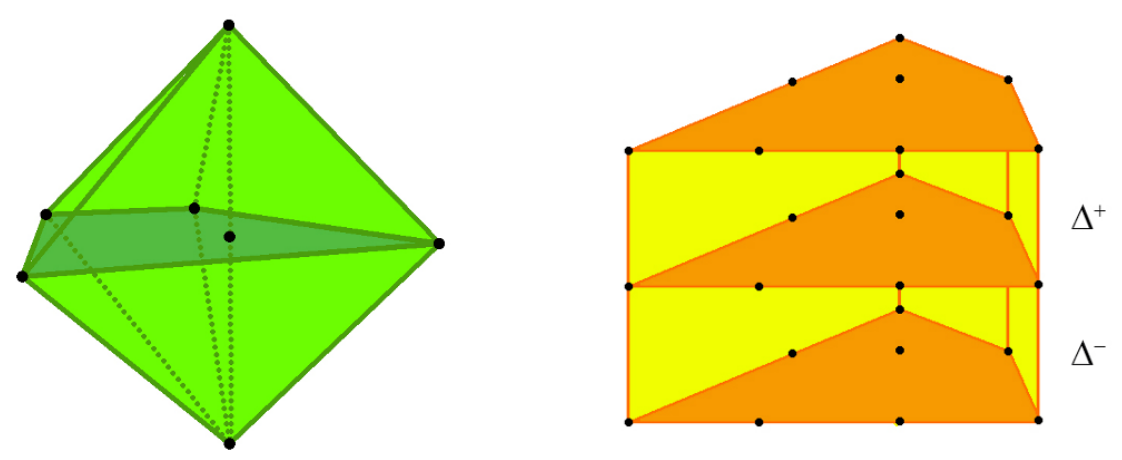

Figure 2. On the left we show the reflexive polytope $\Delta_{3}^{\circ}$, while its dual $\Delta_{3}$ is shown on the right. In this example, the ambient space for the elliptic fiber, specified by $\Delta_{2}^{\circ}$, is given by $\mathrm{Bl}_{1} \mathbb{P}^{(1,1,2)}$.

Here $\left(a_{i} b_{i}\right)$ denote the points of a two-dimensional reflexive polytope $\Delta_{2}^{\circ}$, which will specify the geometry of the elliptic fiber $E$. It is embedded into $\Delta_{3}^{\circ}$ in the $x y$-plane, see the first picture in figure 2. The last column contains the homogeneous coordinate associated to a given vertex. We label the rays of the two-dimensional polytope counter-clockwise by the coordinates $x_{1}, \ldots x_{n}$. In addition, we assign the coordinates $U, V$ to the points $(001)$ and $(00-1)$ which correspond to the rays of the fan of the $\mathbb{P}^{1}$-base. We use the shorthand notation $\mathbb{P}_{[U: V]}^{1}$ to indicate its homogeneous coordinates. Finally, we use the notation $\rho_{H}$ for the ray with corresponding homogeneous coordinate $H$. We denote by $\Sigma_{3}$ the natural simplicial fan associated to $\Delta_{3}^{\circ}$ and denote the corresponding toric variety over the fan of $\Delta^{\circ}$ as $\mathbb{P}_{\Sigma_{3}}$. Provided a fine triangulation of the polytope $\Delta_{3}^{\circ}$ has been chosen, the toric ambient space $\mathbb{P}_{\Sigma_{3}}$ will be Gorenstein and terminal.

A general section $\chi$ of the anti-canonical bundle $\mathcal{O}_{\mathbb{P}_{\Sigma_{3}}}\left(-K_{\mathbb{P}_{\Sigma_{3}}}\right)$ defines a smooth elliptically fibered $\mathrm{K} 3$ surface $X_{2}$. The ambient space of its elliptic fiber $E$ is the toric variety $\mathbb{P}_{\Sigma_{2}}$ that is constructed from the fan $\Sigma_{2}$ of the polytope $\Delta_{2}^{\circ}$ induced by $\Sigma_{3}$. As the toric fibration of $\Sigma_{2}$ over $\Sigma_{\mathbb{P} 1}$ is direct, the section $\chi$ takes the form

$$
\chi=s_{i} \eta^{i} \quad \text { for } \quad s_{i}=s_{i}^{0} U^{2}+s_{i}^{1} U V+s_{i}^{2} V^{2} .
$$

Here $\eta^{i}$ are the sections of the anti-canonical bundle of $\mathcal{O}_{\mathbb{P}_{\Sigma_{2}}}\left(-K_{\mathbb{P}_{\Sigma_{2}}}\right)$, i.e. the range of the index $i$ is given by the number of integral points in $\Delta_{2}$, and $s_{i}^{k}, k=1,2,3$, are constants. Note that, for a very general ${ }^{4} X_{2}$, the dimension $h^{(1,1)}(X)$, of the cohomology group $H^{(1,1)}\left(X_{2}, \mathbb{C}\right)$ can be computed combinatorically from the pair of reflexive polyhedra $\Delta_{3}, \Delta_{3}^{\circ}$ by a generalization of the Batyrev's formula [52]:

$$
h^{(1,1)}(X)=l\left(\Delta^{\circ}\right)-n-1-\sum_{\Gamma^{\circ}} l^{*}\left(\Gamma^{\circ}\right)+\sum_{\Theta^{\circ}} l^{*}\left(\Theta^{\circ}\right) l^{*}\left(\hat{\Theta}^{\circ}\right) .
$$

Here $l(\Delta)\left(l^{*}(\Delta)\right)$ denote the number of (inner) points of the $n$-dimensional polytope $\Delta$. In addition, $\Gamma\left(\Gamma^{\circ}\right)$ denote the codimension one faces of $\Delta\left(\Delta^{\circ}\right)$, while $\Theta$ denotes a codimension two face with $\hat{\Theta}$ being its dual.

\footnotetext{
${ }^{4} \mathrm{~A}$ point is very general if it lies outside a countable union of closed subschemes of positive codimension.
} 


\subsection{Constructing K3 fibrations}

As a next step, we fiber this ambient space over a second $\mathbb{P}_{\left[\lambda_{1}, \lambda_{2}\right]}^{1}$ with homogeneous coordinates $\lambda_{1}, \lambda_{2}$. The following construction is such that the generic fiber consists of a smooth K3 surface $X_{2}$ over a generic point of $\mathbb{P}_{\left[\lambda_{1}, \lambda_{2}\right]}^{1}$ and a split fiber, i.e. a splitting into two half K3 surfaces, over a distinguished point of $\mathbb{P}_{\left[\lambda_{1}, \lambda_{2}\right]}^{1}$, as explained below.

The four-dimensional polytope which describes this construction is given by

$$
\Delta_{4}=\left\{\left(m_{1}, m_{2}, m_{3}, m_{4}\right) \in \mathbb{Z}^{4} \mid\left(m_{1}, m_{2}, m_{3}\right) \in \Delta_{3},-1 \leqslant m_{4} \leqslant 1,\left\{\begin{array}{ll}
m_{4} \geqslant-1 & \text { if } m_{3} \leqslant 0, \\
m_{4} \geqslant m_{3}-1 & \text { if } m_{3} \geqslant 0 .
\end{array}\right\} .\right.
$$

Here, $\Delta_{3}$ denotes the dual polytope of $\Delta_{3}^{\circ}$, cf. the second picture in figure 2 . The faces of $\Delta_{4}$ are given by the (intersection of the) hyperplanes

$$
m_{4}=1, \quad m_{4}=-1, \quad m_{4}=-1+m_{3}, \quad m_{3}=-1, \quad m_{3}=1, \quad \sum_{j=0}^{2} a_{i}^{j} m_{j}=1,
$$

where the last expression is given by the defining hyperplanes of $\Delta_{2}$, the dual of $\Delta_{2}^{\circ}$. We denote by $\Sigma_{4}$ the fan associated to the dual polytope $\Delta_{4}^{\circ}$ of $\Delta_{4}$. In particular, the normal vectors of the facets of $\Delta_{4}$ give the rays of $\Sigma_{4}$. To be explicit, the rays of $\Sigma_{4}$ are given by the rows of the matrix

$$
\left(\begin{array}{cccc|c}
a_{1} & b_{1} & 0 & 0 & x_{1} \\
\vdots & & 0 & 0 & x_{i} \\
a_{n} & b_{n} & 0 & 0 & x_{n} \\
0 & 0 & 1 & 0 & U \\
0 & 0 & 0 & 1 & \lambda_{1} \\
0 & 0 & -1 & 1 & \mu \\
0 & 0 & -1 & 0 & V \\
0 & 0 & 0 & -1 & \lambda_{2}
\end{array}\right) .
$$

We note that the coordinates assigned to its rays as displayed in (3.6) transform as follows under the $\mathbb{C}^{*}$-actions

$$
\left(U: \lambda_{1}: \mu: V: \lambda_{2}\right) \sim\left(a^{-1} U: a b^{-1} \lambda_{1}: a^{-1} b c^{-1} \mu: b^{-1} c V: c^{-1} \lambda_{2}\right)
$$

with $a, b, c \in \mathbb{C}^{*}$.

In analogy to the discussion in the previous section, a section $\chi_{4}$ of the anti-canonical bundle $-K_{\mathbb{P}_{\Sigma_{4}}}$ of the toric variety $\mathbb{P}_{\Sigma_{4}}$ defines a three-dimensional smooth Calabi-Yau manifold $\mathfrak{X}$. In particular, the Calabi-Yau constraint (3.2) generalizes as

$$
\chi_{4}=s_{i} \eta^{i},
$$

where the $\eta^{i}$ are given as before and the coefficients $s_{i}$ now read

$$
\begin{aligned}
s_{i}\left(U, V, \lambda_{1}, \lambda_{2}, \mu\right)= & s_{i}^{1} \lambda_{1} \lambda_{2} U^{2}+s_{i}^{2} \lambda_{1}^{2} \mu U^{2}+s_{i}^{3} \lambda_{2}^{2} U V+s_{i}^{4} \lambda_{1} \lambda_{2} \mu U V+s_{i}^{5} \lambda_{1}^{2} \mu^{2} U V \\
& +s_{i}^{6} \lambda_{2}^{2} \mu V^{2}+s_{i}^{7} \lambda_{1} \lambda_{2} \mu^{2} V^{2}+s_{i}^{8} \lambda_{1}^{2} \mu^{3} V^{2}
\end{aligned}
$$

with constants $s_{i}^{j} \in \mathbb{C}$. 

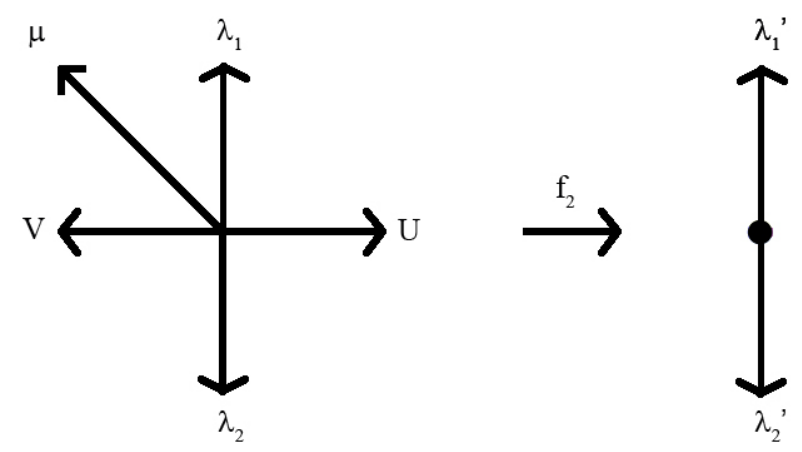

Figure 3. The toric morphism $f_{2}$.

We proceed by observing that the projection on the last two columns in (3.6) yields the polytope $\Delta_{d P_{2}}^{\circ}$ of the toric variety $d P_{2}$, cf. figure 3 . Denoting the fan of $\Delta_{d P_{2}}^{\circ}$ by $\Sigma_{d P_{2}}$ this projection gives rise to a toric map

$$
f_{1}: \Sigma_{4} \longrightarrow \Sigma_{d P_{2}}
$$

In addition, $d P_{2}$ is fibered over the $\mathbb{P}_{\left[\lambda_{1}^{\prime}: \lambda_{2}^{\prime}\right]}^{1}$ as can be seen by projecting onto the fourth column of $\Delta_{4}$, cf. figure 3 , i.e. there is a toric map

$$
f_{2}: \Sigma_{d P_{2}} \longrightarrow \Sigma_{\mathbb{P}^{1}}
$$

where $\Sigma_{\mathbb{P}^{1}}$ is the fan of $\mathbb{P}_{\left[\lambda_{1}^{\prime}: \lambda_{2}^{\prime}\right]}^{1}$. Note that this $\mathbb{P}^{1}$ is isomorphic to $\mathbb{P}_{\left[\lambda_{1}: \lambda_{2}\right]}^{1}$. We denote the composition map of the two by $f=f_{2} \circ f_{1}$.

In summary, we have the following diagram of toric morphisms and induced maps on $\mathfrak{X}$ :

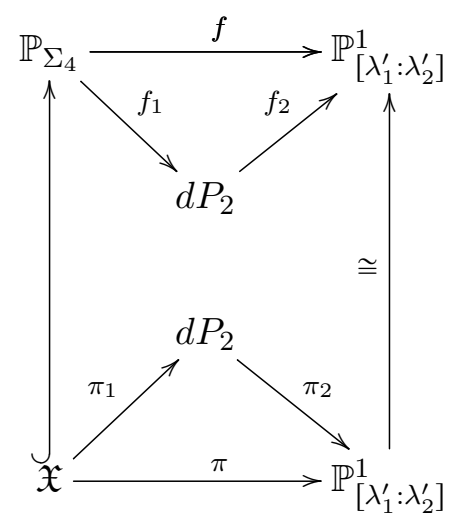

Here we denote the toric maps $f_{1}, f_{2}, f$ and their induced morphisms of toric varieties by the same symbol, respectively. Note that for a generic point, the fiber of $\pi$ is given by a smooth K3 surface $X_{2}$.

In order to prepare for the discussion of the stable degeneration limit, we proceed by discussing the fibration map in more detail. For this purpose, we note the correspondence of facets and rays as displayed in table 3 . The dual $\Delta_{d P_{2}}$ of $\Delta_{d P_{2}}^{\circ}$ with associated monomials is shown in figure 4 . These monomials are the global sections of $K_{d P_{2}}$ and are constructed 


$$
\begin{array}{|c|c|c|}
\hline \text { ray } & \text { facet } & \text { constraint } \\
\rho_{\lambda_{1}} & m_{4}=-1 & s_{\lambda_{1}}=s_{i}^{3} U+s_{i}^{6} \mu \\
\rho_{\mu} & m_{4}=m_{3}-1 & s_{\mu}=s_{i}^{1} \lambda_{1}+s_{i}^{3} V \\
\rho_{\lambda_{2}} & m_{4}=1 & s_{\lambda_{2}}=s_{i}^{2} U^{2}+s_{i}^{5} U V+s_{i}^{8} V^{2} \\
\hline
\end{array}
$$

Table 3. The correspondence between the rays of $\Delta_{d P_{2}}^{\circ}$ and the facets of $\Delta_{d P_{2}}$. The last column displays the global sections that embed the associated divisor into $\mathbb{P}^{1}$ and $\mathbb{P}^{2}$, respectively. The coefficients on the right-hand side refer to equation (3.9).

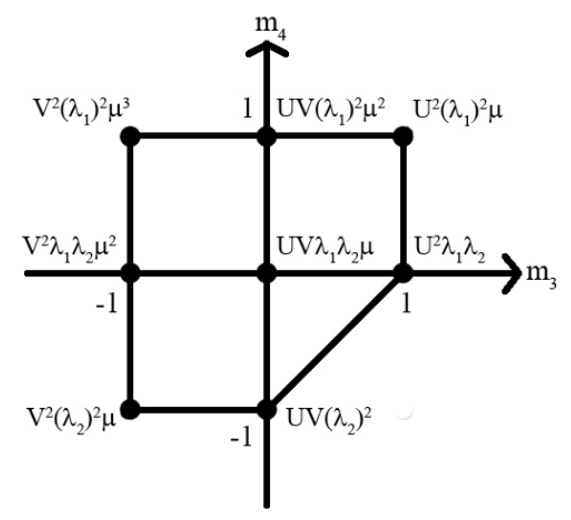

Figure 4. The dual polytope $\Delta_{d P_{2}}$ and the associated monomials.

according to $[53]$

$$
\chi_{d P_{2}}=\sum_{P \in \Delta_{d P_{2}}} \prod_{P^{*} \in \Delta_{d P_{2}}^{*}} a_{P} x_{P^{*}}^{\left\langle P, P^{*}\right\rangle+1} .
$$

Here $x_{P^{*}}$ denotes the coordinate which is associated to the corresponding ray of the toric diagram and $a_{P}$ are constants. By the correspondence between cones of $\Delta_{d P_{2}}^{\circ}$ and vertices of $\Delta_{d P_{2}}$ the vertex corresponding to the monomial $V^{2} \lambda_{2}^{2} \mu$ is dual to the cone spanned by the rays $\rho_{U}$ and $\rho_{\lambda_{1}}$. We denote the coordinates associated to the two rays of $\mathbb{P}_{\left[\lambda_{1}: \lambda_{2}\right]}^{1}$ inside $\Delta_{d P_{2}}^{0}$ appearing in (3.11) by $\rho_{\lambda_{1}^{\prime}}$ and $\rho_{\lambda_{2}^{\prime}}$. Note that $f_{2}^{-1}\left(\rho_{\lambda_{1}^{\prime}}\right)=\left\{\rho_{\lambda_{1}}, \rho_{\mu}\right\}$, while $f_{2}^{-1}\left(\rho_{\lambda_{2}^{\prime}}\right)=\left\{\rho_{\lambda_{2}}\right\}$.

\subsection{The toric stable degeneration limit}

In the following, we aim to show that the general fiber of the map $\pi$ gives rise to a smooth K3 surface while the pre-image of the point $\left[\lambda_{1}^{\prime}: \lambda_{2}^{\prime}\right]=[1: 0]$ gives rise to a degeneration into two half K3 surfaces $X_{2}^{ \pm}$that intersect in the elliptic fiber $Z_{1}$ over the point of intersection of the two $\mathbb{P}^{1}$ which are the respective bases of their elliptic fibrations.

Let us first consider the toric variety $f_{2}^{-1}\left(\lambda_{2}^{\prime}=0\right)$ corresponding to the pre-image in $\Delta_{d P_{2}}^{0}$ of $\rho_{\lambda_{2}^{\prime}}$. It is given by the star of $\rho_{\lambda_{2}}$ in $\Delta_{d P_{2}}^{0}$ which is just the generic fiber of $f_{2}$. Indeed, if $\lambda_{2}=0$, the coordinates $\mu$ and $\lambda_{1}$ are non-vanishing due to the Stanley-Reisner ideal. Two of the scaling relations (3.7) can be used in order to eliminate the latter two variables while the remaining (linear combination) endows the coordinates $U, V$ with the well-known scaling relations of $\mathbb{P}_{[U, V]}^{1}$. In addition, the monomials associated to the vertices 
of the dual facet of $\rho_{\lambda_{2}}$ give rise to the following sections

$$
s_{\lambda_{2}}:=s_{i}^{2} U^{2}+s_{i}^{5} U V+s_{i}^{8} V^{2},
$$

as follows from (3.9) by setting $\lambda_{2}=0$. These provide precisely the global sections of $O_{\mathbb{P}^{1}}(2)$ that are needed for the Veronese embedding, i.e. the embedding of $\mathbb{P}_{[U, V]}^{1}$ into $\mathbb{P}^{2}$ as a conic

$$
[U: V] \longmapsto\left[U^{2}: U V: V^{2}\right]
$$

In contrast, the preimage of $\rho_{\lambda_{1}^{\prime}}$ consists of the two divisors $\lambda_{1}=0$ and $\mu=0$. In this case the Stanley-Reisner ideal forbids the vanishing of the coordinates $V, \lambda_{2}$ and $U, \lambda_{2}$ respectively. Taking again into account the scaling relations (3.7), one observes that the pre-image of the divisor $\lambda_{1}^{\prime}=0$ consists of two $\mathbb{P}^{1}$ 's that are given by

$$
D_{\lambda_{1}}=[U: 0: \mu: 1: 1], \quad D_{\mu}=\left[1: \lambda_{1}: 0: V: 1\right] .
$$

These intersect in precisely one point given by $[1: 0: 0: 1: 1]$. One identifies the dual facets of $\rho_{\lambda_{1}}$ and $\rho_{\mu}$ as $m_{4}=-1$ and $m_{4}=m_{3}-1$. In this case the global sections are given by

$$
s_{\lambda_{1}}:=s_{i}^{3} U+s_{i}^{6} \mu, \quad s_{\mu}:=s_{i}^{1} \lambda_{1}+s_{i}^{3} V,
$$

as follows again from (3.9). This induces in this case only the trivial embedding via the identity map. Note that the union of the two divisors $D_{\lambda_{1}}$ and $D_{\mu}$ is given by a degenerate conic

$$
z_{1} z_{3}=z_{2}^{2} \lambda_{1} \mu, \quad \text { with } \quad\left(V^{2} \mu, U V, U^{2} \lambda_{1}\right) \mapsto\left[z_{1}: z_{2}: z_{3}\right] \in \mathbb{P}^{2},
$$

which splits as just observed into the two lines $z_{1}=0, z_{3}=0$ at $\lambda_{1}=0$ and $\mu=0$.

A similar reasoning applies to the pre-image of $\rho_{\lambda_{1}^{\prime}}$ under the composite map $f$. As noted above, we have $f^{-1}\left(\rho_{\lambda_{1}^{\prime}}\right)=\left\{\rho_{\mu}, \rho_{\lambda_{1}}\right\}$, which implies that the pre-image is given by the two divisors $\mathbb{P}_{\Sigma_{3}^{+}}=\{\mu=0\}$ and $\mathbb{P}_{\Sigma_{3}^{-}}=\left\{\lambda_{1}=0\right\}$ in $\mathbb{P}_{\Sigma_{4}}$. They are obtained as the star of $\rho_{\mu}$ and $\rho_{\lambda_{1}}$ in $\Delta_{4}^{0}$, respectively, with their fans $\Sigma_{3}^{ \pm}$induced by $\Sigma_{4}$. The corresponding respective dual facets are given by the three-dimensional facets $m_{4}=-1$ and $m_{4}=m_{3}-1$ in $\Delta_{4}$. In addition, this gives rise to a splitting of $\Delta_{3}$ as

$$
\begin{aligned}
& \Delta_{3}^{+}=\left\{\left(m_{1}, m_{2}, m_{3}\right) \in \mathbb{Z}^{3} \mid\left(m_{1}, m_{2}\right) \in \Delta_{2}, m_{3} \in\{0,1\}\right\}, \\
& \Delta_{3}^{-}=\left\{\left(m_{1}, m_{2}, m_{3}\right) \in \mathbb{Z}^{3} \mid\left(m_{1}, m_{2}\right) \in \Delta_{2}, m_{3} \in\{-1,0\}\right\},
\end{aligned}
$$

which is also referred to as the top and bottom splitting [10], cf. figure 2. Thus, the section of the anti-canonical bundle $\mathcal{O}\left(-K_{\mathbb{P}_{\Sigma_{3}}}\right)$ in (3.2) in the limit becomes the sum of

$$
\begin{aligned}
& \chi_{X_{2}^{+}}:=s_{i}^{+} \eta^{i}, \quad \text { with } \quad s_{i}^{+}=s^{+0}{ }_{i}^{0} V+s^{+1}{ }_{i}^{1} \lambda_{1}, \\
& \chi_{X_{2}^{-}}:=s_{i}^{-} \eta^{i}, \quad \text { with } \quad s_{i}^{-}=s^{-0}{ }_{i}^{0} U+s^{-1}{ }_{i}^{1} \mu,
\end{aligned}
$$

so that we can define the two surfaces $X_{2}^{ \pm}$as

$$
X_{2}^{+}=\left.X_{2}\right|_{\mathbb{P}_{\Sigma_{3}^{+}}}=\{\chi=\mu=0\}, \quad X_{2}^{-}=\left.X_{2}\right|_{\mathbb{P}_{\Sigma_{3}^{-}}}=\left\{\chi=\lambda_{1}=0\right\} .
$$


As we will prove in the next subsection, $X_{2}^{+}$and $X_{2}^{-}$are two rational elliptic surfaces (half K3 surfaces).

In contrast, the pre-image of $\rho_{\lambda_{2}^{\prime}}$ is given by the whole three-dimensional fan $\Sigma_{3}$ as it is also for a generic point in $\mathbb{P}_{\left[\lambda_{1}: \lambda_{2}\right]}^{1}$. To justify the latter statement inspect the fiber above the origin 0 of the fan $\Sigma_{\mathbb{P}^{1}}$ corresponding to a generic point in $\mathbb{P}_{\left[\lambda_{1}: \lambda_{2}\right]}^{1}$.

Finally, we remark that the two rational elliptic surfaces $X_{2}^{+}, X_{2}^{-}$that arise at the loci $\{\mu=0\}$ and $\{\mu=0\}$, respectively, are independent of the K3 surface which appears over the locus $\left\{\lambda_{2}=0\right\}$. In the following, we explain how the half K3 surfaces can be obtained from the data of the K3 surface $X_{2}$ directly. As the notation used so far is rather heavy, which is unfortunately necessary, we introduce a slightly easier notation that will be used in the discussion of explicit examples in section 4 . We rewrite a general hypersurface constraint as

$$
\chi=s_{i} \eta^{i}, \quad s_{i}=s_{i 1} U^{2}+s_{i 2} U V+s_{i 3} V^{2},
$$

which requires, depending on the situation at hand, the following identifications between the coefficients of (3.22) and of (3.9):

$$
\begin{aligned}
& s_{i 1} \equiv s_{i}^{2}, \quad s_{i 2} \equiv s_{i}^{5}, \quad s_{i 3} \equiv s_{i}^{8}, \\
& \text { or } \quad s_{i 1} \equiv s_{i}^{1}, \quad s_{i 2} \equiv s_{i}^{3}, \quad s_{i 3} \equiv s_{i}^{6} \text {. }
\end{aligned}
$$

However, it is crucial to note that the pair of coordinates $U, V$ is only suited to describe the base $\mathbb{P}^{1}$ of the K3 surface $X_{2}$, while the base coordinates $\mathbb{P}^{1}$ 's of $X_{2}^{+}$and $X_{2}^{-}$are given by $\lambda_{1}, V$ and $U, \mu$, respectively.

\subsection{Computing the canonical classes of the half K3 surfaces $X_{2}^{ \pm}$}

In this subsection, we discuss how the half $\mathrm{K} 3$ surfaces $X_{2}^{ \pm}$can be re-discovered in the toric stable degeneration limit. Note that the two components $\mathbb{P}_{\Sigma_{3}^{+}}$and $\mathbb{P}_{\Sigma_{3}^{-}}$of the degenerate fiber, as divisors in $\mathbb{P}_{\Sigma_{4}}$, should equal the generic fiber $\mathbb{P}_{\Sigma_{3}}$ :

$$
\mathbb{P}_{\Sigma_{3}^{+}}+\mathbb{P}_{\Sigma_{3}^{-}} \cong \mathbb{P}_{\Sigma_{3}}
$$

In addition, we have

$$
\mathbb{P}_{\Sigma_{3}} \cdot \mathbb{P}_{\Sigma_{3}^{ \pm}}=0
$$

as the generic fiber can be moved away from the locus $\lambda_{1}^{\prime}=0$, cf. figure 3 . This allows us to compute the canonical bundle of $\mathbb{P}_{\Sigma_{3}^{ \pm}}$using adjunction in $\mathbb{P}_{\Sigma_{4}}$ as

$$
K_{\mathbb{P}_{\Sigma_{3}}^{ \pm}}=\left.\left(K_{\mathbb{P}_{\Sigma_{4}}} \otimes \mathcal{O}_{\mathbb{P}_{\Sigma_{4}}}\left(\mathbb{P}_{\Sigma_{3}^{ \pm}}\right)\right)\right|_{\mathbb{P}_{\Sigma_{3}}^{ \pm}}=\left.K_{\mathbb{P}_{\Sigma_{4}}}\right|_{\mathbb{P}_{\Sigma_{3}}^{ \pm}} \otimes \mathcal{O}_{\mathbb{P}_{\Sigma_{3}}^{ \pm}}\left(-\mathbb{P}_{\Sigma_{3}^{ \pm}} \cdot \mathbb{P}_{\Sigma_{3}^{\mp}}\right),
$$

where we used (3.24) and (3.25). Note that the divisor corresponding to the last term equals the class of the ambient space $\mathbb{P}_{\Sigma_{2}}$ of elliptic fiber of $X_{2}$, i.e. $\mathbb{P}_{\Sigma_{3}^{+}} \cdot \mathbb{P}_{\Sigma_{3}^{-}}=\mathbb{P}_{\Sigma_{2}}$. Making one more time use of the adjunction formula, one finally arrives at

$$
\begin{aligned}
K_{X_{2}^{ \pm}} & =\left.\left(K_{\mathbb{P}_{\Sigma_{3}^{ \pm}}} \otimes \mathcal{O}_{\mathbb{P}_{\Sigma_{3}^{ \pm}}}\left(X_{2}^{ \pm}\right)\right)\right|_{X_{2}^{ \pm}}=\left.\left(\left.K_{\mathbb{P}_{\Sigma_{4}}}\right|_{\mathbb{P}_{\Sigma_{3}}^{ \pm}} \otimes \mathcal{O}_{\mathbb{P}_{\Sigma_{3}}^{ \pm}}\left(-\mathbb{P}_{\Sigma_{2}}\right) \otimes \mathcal{O}_{\mathbb{P}_{\Sigma_{3}^{ \pm}}}\left(X_{2}^{ \pm}\right)\right)\right|_{X_{2}^{ \pm}} \\
& =\left.\mathcal{O}_{\mathbb{P}_{\Sigma_{3}}^{ \pm}}\left(-\mathbb{P}_{\Sigma_{2}}\right)\right|_{X^{ \pm}}=\mathcal{O}_{X_{2}^{ \pm}}(-\mathcal{E}),
\end{aligned}
$$


where we used (3.26) in the second equality and $\left.K_{\mathbb{P}_{\Sigma_{4}}}\right|_{\mathbb{P}_{\Sigma_{3}}^{ \pm}}=\mathcal{O}_{\mathbb{P}_{\Sigma_{3}^{ \pm}}}\left(X_{2}^{ \pm}\right)$. Thus, the anticanonical class of $X_{2}^{ \pm}$is given by that of the elliptic fiber $E$ which leads to the conclusion that $X_{2}^{ \pm}$is indeed a rational elliptic surface.

\section{Examples of heterotic/F-theory duals with U(1)'s}

In the section, we use the tools of section 3 to construct explicit elliptically fibered CalabiYau two- and threefolds whose stable degeneration limit is well under control. Our geometries have generically two sections, which give rise to a $\mathrm{U}(1)$-factor in the corresponding F-theory compactification. Performing the toric symplectic cut allows us to explicitly track these sections through the stable degeneration limit and to make non-trivial statements about the vector bundle data on the heterotic side in which the U(1)-factor in the effective theory is encoded. Finally, after having performed the stable degeneration limit as discussed in section 3.3, we split the resulting half K3 surfaces into the spectral cover polynomial and the constraint for the heterotic elliptic curve. Then, we determine the common solutions of the latter two constraints which encode the data of a (split) spectral cover. The general geometries we consider as well as the procedure we apply is discussed in section 4.1. Despite the fact that we do not determine the embedding of the structure group into $\mathrm{E}_{8}$ directly, we are able to match the spectral cover with the resulting gauge group in all cases. In particular, we consider three different classes of examples. In subsection 4.2 we investigate a number of examples whose heterotic dual gives rise to a split spectral cover. This class of examples has generically one $U(1)$ factor embedded into both $E_{8}$-bundles of which only a linear combination is massless. The next class of examples considered in subsection 4.3 displays torsional points in its spectral cover. There is one example with a $\mathrm{U}(1)$-factor on the F-theory side which is found to be only embedded into one $\mathrm{E}_{8}$-bundle while the other $\mathrm{E}_{8}$-bundle is kept intact. Finally, in the last subsection 4.4 we consider an example where the structure group reads $\mathrm{SU}(2) \times \mathrm{SU}(3)$. However, we argue that it is embedded in such a way that its centralizer necessarily contains a $U(1)$ factor.

\subsection{The geometrical set-up: toric hypersurfaces in $\mathbb{P}^{1} \times \mathrm{Bl}_{1} \mathbb{P}^{(1,1,2)}$}

For convenience, we recall the three-dimensional polyhedron $\Delta_{3}^{\circ}$ for the resolved toric ambient space $\mathbb{P}_{\Sigma_{3}}=\mathbb{P}^{1} \times \mathrm{Bl}_{1} \mathbb{P}^{(1,1,2)}$. It is given by the points

$$
\left(\begin{array}{ccc|c}
-1 & 1 & 0 & x_{1} \\
-1 & -1 & 0 & x_{2} \\
1 & 0 & 0 & x_{3} \\
0 & 1 & 0 & x_{4} \\
-1 & 0 & 0 & x_{5} \\
0 & 0 & 1 & U \\
0 & 0 & -1 & V
\end{array}\right)
$$

Here, $x_{1}, \ldots x_{5}$ are homogeneous coordinates on the resolved variety $\mathrm{Bl}_{1} \mathbb{P}^{(1,1,2)}$, while $U, V$ denote the two homogeneous coordinates of $\mathbb{P}^{1}$. In particular, $x_{5}$ resolves the $A_{1}$-singularity of the space $\mathrm{Bl}_{1} \mathbb{P}^{(1,1,2)}$. 
A generic section of the anti-canonical bundle of the ambient space $\mathbb{P}_{\Sigma_{3}}$ takes the form

$$
\begin{aligned}
\chi:= & s_{1} x_{1}^{4} x_{4}^{3} x_{5}^{2}+s_{2} x_{1}^{3} x_{2} x_{4}^{2} x_{5}^{2}+s_{3} x_{1}^{2} x_{2}^{2} x_{4} x_{5}+s_{4} x_{1} x_{2}^{3} x_{5}^{2}+s_{5} x_{1}^{2} x_{3} x_{4}^{2} x_{5} \\
& +s_{6} x_{1} x_{2} x_{3} x_{4} x_{5}+s_{7} x_{2}^{2} x_{3} x_{5}+s_{8} x_{3}^{2} x_{4}=0,
\end{aligned}
$$

where the coefficients $s_{i}$ are homogeneous quadratic polynomials in $U, V$. An elliptically fibered K3 surface is defined by $X_{2}=\{\chi=0\}$. As can be seen for example in its Weierstrass form, the K3 surface generically has a Kodaira fiber of type $\mathrm{I}_{2}$ at the locus $s_{8}=0$. It is resolved by the divisor $\left\{x_{5}=0\right\} \cap X_{2}$ as mentioned above. ${ }^{5}$ In addition, $X_{2}$ generically has a Mordell-Weil group of rank one. A choice of zero section $S_{0}$ and generator of the Mordell-Weil group $S_{1}$ are given by

$$
S_{0}=X_{2} \cap\left\{x_{1}=0\right\}, \quad S_{1}=X_{2} \cap\left\{x_{4}=0\right\} .
$$

Explicitly, their coordinates read

$$
S_{0}=\left\{\begin{array}{ll}
{\left[0: 1: 1: s_{7}:-s_{8}\right]} & \text { generically }, \\
{[0: 1: 1: 0: 1]} & \text { if } s_{7}=0, \\
{[0: 1: 1: 1: 0]} & \text { if } s_{8}=0,
\end{array} \quad S_{1}= \begin{cases}{\left[s_{7}: 1:-s_{4}: 0: 1\right]} & \text { generically } \\
{[0: 1: 1: 0: 1]} & \text { if } s_{7}=0 \\
{[1: 1: 0: 0: 1]} & \text { if } s_{4}=0\end{cases}\right.
$$

Here we distinguished the special cases with $s_{7}=0$ and $s_{8}=0$, respectively, from the generic situation. Using the fact that the generic K3 surface $X_{2}$ has $h^{(1,1)}=5$ [52], we, hence, conclude that the full F-theory gauge group $G_{X_{2}}$ is ${ }^{6}$

$$
G_{X_{2}}=\mathrm{SU}(2) \times \mathrm{SU}(2) \times \mathrm{U}(1) .
$$

We note that if $s_{7}=0$, one observes that the two sections coincide, as was also employed in [54]. That the converse is true is shown in appendix C. This is expected as the vanishing of $s_{7}$ can be interpreted as a change of the toric fibre ambient space from $\mathrm{Bl}_{1} \mathbb{P}^{(1,1,2)}$ to $\mathbb{P}^{(1,2,3)}$, which has a purely non-Abelian gauge group [30].

\subsubsection{Engineering gauge symmetry: specialized sections of $-K_{\mathbb{P}^{1} \times \mathrm{Bl}_{1} \mathbb{P}^{(1,1,2)}}$}

In order to construct examples with higher rank gauge groups, we tune the coefficients of $\chi$ further. To be concrete, every $s_{i}$ in (4.2) takes the form

$$
s_{i}=s_{i 1} U^{2}+s_{i 2} U V+s_{i 3} V^{2}
$$

and a specialization corresponds to the identical vanishing of some $s_{i j}$. This specialization of coefficients implies that $\Delta$, the dual polyhedron of $\mathbb{P}^{1} \times \mathrm{Bl}_{1} \mathbb{P}^{(1,1,2)}$, gets replaced by the Newton polytope $\Delta_{\text {spec. }}$ of the specialized constraint, compare also figure 5 . As a technical side-remark we note that we strictly speaking refer with $\Delta_{\text {spec. }}$ to the convex

\footnotetext{
${ }^{5}$ As the details of the resolution are not important, we can set $x_{5}=1$ in most computations performed here.

${ }^{6}$ We note that $s_{8}=0$ has two solutions on $\mathbb{P}^{1}$. If we consider higher dimensional bases of the elliptic fibration, we will just have one $\mathrm{SU}(2)$ factor as $s_{8}=0$ is in general an irreducible divisor.
} 

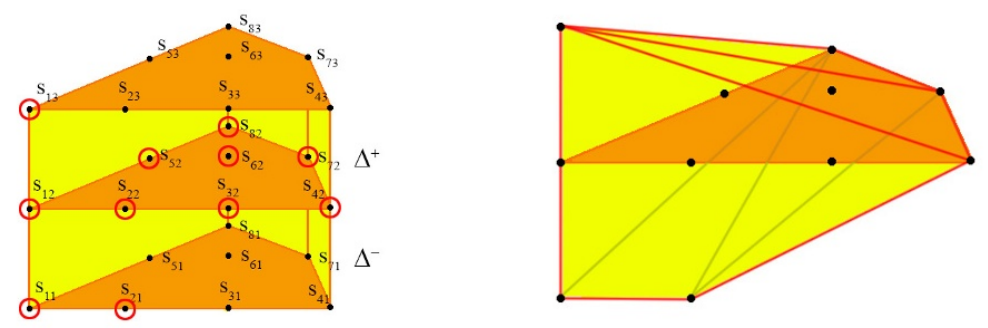

Figure 5. This figure illustrates a specialization of the coefficients of the hypersurface $\chi=0$ such that the resulting gauge group is enhanced to $\mathrm{E}_{7} \times \mathrm{E}_{6} \times \mathrm{U}(1)$, see also the discussion in section 4.2.2. In the left picture, the non-vanishing coefficients are marked by a circle in the polytope $\Delta_{3}$. In the right figure the new polytope, i.e. the Newton polytope of the specialized constraint $\chi=0$, is shown.

hull of the points defined by the non-vanishing monomials in (4.6) respectively (4.2). As a consequence, also $\Delta^{\circ}$ changes to the dual of $\Delta_{\text {spec. }}^{\circ}$. Thus, we have secretly changed the toric ambient space by this specialization of coefficients. It is crucial to note that only those specializations are allowed whose corresponding specialized polyhedron $\Delta_{\text {spec. }}$ is reflexive. The latter condition is equivalent to the associated toric variety being Gorenstein and Fano. Set-ups which are not reflexive can in particular have ill-defined stable-degeneration limits within our formalism.

In order to determine the gauge group of this specialized hypersuface, we need to transform $\chi=0$ into its corresponding Tate or Weierstrass normal form. For convenience, we provide the Weierstrass as well as the Tate form of the most general hypersurface in appendix A.

\subsubsection{Stable degeneration and the spectral cover polynomial}

As a next step, we show how the K3 surface $X_{2}$ defined via (4.2) can be decomposed into the two half K3 surfaces $X_{2}^{ \pm}$and the heterotic elliptic curve as well as the two spectral cover polynomials, respectively. First, we write the Calabi-Yau hypersurface equation (4.3) for $X_{2}$ as

$$
\chi=p^{+}\left(x_{i}, s_{j 1}\right) U^{2}+p_{0}\left(x_{i}, s_{j 2}\right) U V+p^{-}\left(x_{i}, s_{j 3}\right) V^{2},
$$

for appropriate polynomials $p^{+}, p_{0}$ and $p^{-}$depending on the fiber coordinates $x_{i}$. By the results of the previous section 3.3, the K3 surface $X_{2}$ in the semistable degeneration limit can be described by the half K3 surfaces $X_{2}^{ \pm}$. Practically, $X_{2}^{+}$arises by setting the term $V^{2}$ in (4.7) to zero and factoring out $U$ and vice versa, $X^{-}$is obtained by setting $U^{2}$ to zero and factoring out $V$. In conclusion, the defining equations for $X_{2}^{ \pm}$are given as

$$
X_{2}^{+}: \quad p^{+}\left(x_{i}, s_{j 1}\right) U+p_{0}\left(x_{i}, s_{j 2}\right) V=0, \quad X_{2}^{-}: \quad p^{-}\left(x_{i}, s_{j 3}\right) V+p_{0}\left(x_{i}, s_{j 2}\right) U=0 .
$$

However, it is crucial to note that this is just a shortcut to obtain the defining equations for $X_{2}^{ \pm}$and that $U, V$ are not the right coordinates for the base $\mathbb{P}^{1}$ of the two rational elliptic surfaces $X_{2}^{ \pm}$. As explained in more detail in section (4.7), $X^{+}$is coordinized by $V, \lambda_{1}$ and $X^{-}$is coordinized by $U, \mu$. Thus, the correct form of the defining constraint for 
$X_{2}^{+}$is given by

$$
X_{2}^{+}: \quad p^{+}\left(x_{i}, s_{j 1}\right) \lambda_{1}+p_{0}\left(x_{i}, s_{j 2}\right) V=0, \quad X_{2}^{-}: \quad p^{-}\left(x_{i}, s_{j 3}\right) \mu+p_{0}\left(x_{i}, s_{j 2}\right) U=0 .
$$

It follows that generically the two linearly independent sections (4.4) of the K3 become independent sections in the half K3s, which we denote, by abuse of notation, by the same symbols. They intersect along the common (heterotic) elliptic curve. This is a novel property of our toric degeneration.

In addition, the heterotic elliptic curve is given as $p_{0}\left(x_{i}, s_{j}\right)=0$ while the data of the two background bundles are given by the spectral cover polynomials $p^{+}\left(x_{i}, s_{j 1}\right)=0$ and $p^{-}\left(x_{i}, s_{j 3}\right)=0$. The structure group of the two heterotic bundles is then determined by the common solutions of $p_{0}\left(x_{i}, s_{j}\right)=0$ with $p^{ \pm}\left(x_{i}, s_{j 1 / 3}\right)=0$ using the results and techniques from section 2.2 .

\subsubsection{Promotion to elliptically fibered threefolds}

Eventually, we are interested in examples of six-dimensional heterotic/F-theory duality. In order to promote the $\mathrm{K} 3$ surfaces $X_{2}$ constructed above to elliptically fibered threefolds we promote the coefficients $s_{i j}$, defined in (4.6), to sections of a line bundle of another $\mathbb{P}^{1}$ with homogeneous coordinates $R, T$. The base of the previously considered K3 surface and the new $\mathbb{P}^{1}$ form a Hirzebruch surface $\mathbb{F}_{n}$. At this point, we only consider base geometries which are Fano and restrict our discussion to $\mathbb{F}_{0}$ and $\mathbb{F}_{1}$ for simplicity, avoiding additional singularities in the heterotic elliptic fibration. For these two geometries, the explicit form of the $s_{i j}$ reads

$$
s_{i j}=s_{i j 1} R^{2}+s_{i j 2} R T+s_{i j 3} T^{2}
$$

for $\mathbb{F}_{0}$ and

$$
s_{i}=s_{i 11} R+s_{i 12} T+s_{i 21} R^{2}+s_{i 22} R T+s_{i 23} T^{2}+s_{i 31} R^{3}+s_{i 32} R^{2} T+s_{i 33} R T^{2}+s_{i 34} T^{3},
$$

for the geometry $\mathbb{F}_{1}$.

Next, we observe that the explicit expression of the discriminant of the heterotic CalabiYau manifold $Z_{n}$, which is given by $p_{0}=0$, contains a factor of $s_{82}^{2}$. While this is certainly not a problem in $8 \mathrm{D}$, as $s_{82}$ is just a constant there, it gives rise to an $\mathrm{SU}(2)$-singularity at co-dimension one in the heterotic K3 surface $Z_{2}$. This can be cured by a resolution of this singularity through an exceptional divisor $E$, which is the analog of $x_{5}$ in (4.1). In particular, the solutions to the spectral cover constraint will pass through the singular point in the fiber. Thus, one expects that the spectral cover curve will pick up contributions from the class $E$ in general. A similar situation has been analyzed in [55] where it has been argued that the introduction of this exceptional divisor will not change the structure of the spectral cover as an $N$-sheeted branched cover of the base except for a finite number of points where it wraps a whole new fiber component over the base. As discussed in [55], this introduces more freedom in the construction of the heterotic vector bundle $V$. As this work focuses on the mapping of U(1)-factors under the heterotic/F-theory duality, we only concentrate on the generic structure of the spectral cover and leave the resolution of this singularity as well as an exploration of the freedom in the construction of $V$ to future works. 


\subsection{U(1)'s arising from U(1) factors in the heterotic structure group}

In this section, we consider examples that have an additional rational section in the dual heterotic geometry. We consider K3 surfaces in F-theory, which are given as hypersurfaces in $\mathrm{Bl}_{1} \mathbb{P}^{(1,1,2)} \times \mathbb{P}^{1}$ with appropriately specialized coefficients generating a corresponding gauge symmetry. Elliptic K3 fibered Calabi-Yau threefolds are constructed straightforwardly as described in section 4.1.3. Thus, our following discussion will be equally valid in 6D, although, in order to avoid confusion, we present our geometric discussions in 8D. Having this in mind we, therefore, drop here in the rest of this work the subscripts on all considered manifolds $X_{n+1}, X_{n+1}^{ \pm}$and $Z_{n}$, respectively. In the following, we discuss the main geometric properties of the Calabi-Yau manifold $X$, demonstrate heterotic/F-theory duality and relations among different examples by a chain of Higgsings.

We begin by a summary of key results and by setting some notation. As we will see, all considered examples have the same heterotic Calabi-Yau manifold $Z$ in common. It is given by the most generic section of the anti-canonical bundle in $\mathrm{Bl}_{1} \mathbb{P}^{(1,1,2)}$ reading

$Z: \quad s_{12} x_{1}^{4}+s_{22} x_{1}^{3} x_{2}+s_{32} x_{1}^{2} x_{2}^{2}+s_{42} x_{1} x_{2}^{3}+s_{52} x_{1}^{2} x_{3}+s_{62} x_{1} x_{2} x_{3}+s_{72} x_{2}^{2} x_{3}+s_{82} x_{3}^{2}=0$.

Here and in the following, we refer to the $\mathbb{P}^{(1,1,2)}$ coordinates only and work with the limit $x_{4} \rightarrow 1, x_{5} \rightarrow 1$.

The examples considered in the following only differ among each other by the spectral covers, i.e. by the choice of the coefficients $s_{i 1}$ and $s_{i 3}$ in (3.22), which will be different in each case. Generically, all examples will have a U(1)-factor embedded into the structure groups of both heterotic vector bundles $V_{1}, V_{2}$. Thus, the maximal non-Abelian gauge group determining any chain of Higgsings is given by $E_{7} \times E_{7}$. For later reference we also note the Weierstrass normal form of (4.12) is given by

$$
\begin{aligned}
W_{Z}: y^{2}=x^{3}+ & \left(-\frac{1}{48} s_{62}^{4}+\frac{1}{6} s_{52} s_{62}^{2} s_{72}-\frac{1}{3} s_{52}^{2} s_{72}^{2}-\frac{1}{2} s_{42} s_{52} s_{62} s_{82}+\frac{1}{6} s_{32} s_{62}^{2} s_{82}\right. \\
& \left.+\frac{1}{3} s_{32} s_{52} s_{72} s_{82}-\frac{1}{2} s_{22} s_{62} s_{72} s_{82}+s_{21} s_{72}^{2} s_{82}-\frac{1}{3} s_{32}^{2} s_{82}^{2}+s_{22} s_{42} s_{82}^{2}\right) x \\
+ & \left(\frac{1}{864} s_{62}^{6}-\frac{1}{72} s_{52} s_{62}^{4} s_{72}+\frac{1}{18} s_{52}^{2} s_{62}^{2} s_{72}^{2}-\frac{2}{27} s_{52}^{3} s_{72}^{3}+\frac{1}{24} s_{42} s_{52} s_{62}^{3} s_{82}\right. \\
& -\frac{1}{72} s_{32} s_{62}^{4} s_{82}-\frac{1}{6} s_{42} s_{52}^{2} s_{62} s_{72} s_{82}+\frac{1}{36} s_{32} s_{52} s_{62}^{2} s_{72} s_{82}+\frac{1}{24} s_{22} s_{62}^{3} s_{72} s_{82} \\
& +\frac{1}{9} s_{32} s_{52}^{2} s_{72}^{2} s_{82}-\frac{1}{6} s_{22} s_{52} s_{62} s_{72}^{2} s_{82}-\frac{1}{12} s_{21} s_{62}^{2} s_{72}^{2} s_{82}+\frac{1}{3} s_{21} s_{52} s_{72}^{3} s_{82} \\
& +\frac{1}{4} s_{42}^{2} s_{52}^{2} s_{82}^{2}-\frac{1}{6} s_{32} s_{42} s_{52} s_{62} s_{82}^{2}+\frac{1}{18} s_{32}^{2} s_{62}^{2} s_{82}^{2}-\frac{1}{12} s_{22} s_{42} s_{62}^{2} s_{82}^{2} \\
& +\frac{1}{9} s_{32}^{2} s_{52} s_{72} s_{82}^{2}-\frac{1}{6} s_{22} s_{42} s_{52} s_{72} s_{82}^{2}-\frac{1}{6} s_{22} s_{32} s_{62} s_{72} s_{82}^{2}+s_{21} s_{42} s_{62} s_{72} s_{82}^{2} \\
& \left.+\frac{1}{4} s_{22}^{2} s_{72}^{2} s_{82}^{2}-\frac{2}{3} s_{21} s_{32} s_{72}^{2} s_{82}^{2}-\frac{2}{27} s_{32}^{3} s_{82}^{3}+\frac{1}{3} s_{22} s_{32} s_{42} s_{82}^{3}-s_{21} s_{42}^{2} s_{82}^{3}\right) .
\end{aligned}
$$

In the stable degeneration limit the heterotic geometry $Z$ generically inherits two sections $S_{0}^{Z}, S_{1}^{Z}$ from the elliptically fibered K3 defined in (4.2). These are the zero section as well 
as the generator of the $\mathrm{U}(1)$ and are in Weierstrass normal form explictly given as

$$
\begin{aligned}
S_{0}^{Z}= & {[1: 1: 0] } \\
S_{1}^{Z}= & {\left[\frac{1}{12}\left(s_{62}^{2} s_{72}^{2}-4 s_{52} s_{72}^{3}-12 s_{42} s_{62} s_{72} s_{82}+8 s_{32} s_{72}^{2} s_{82}+12 s_{42}^{2} s_{82}^{2}\right): \frac{1}{2} s_{82}\left(s_{42} s_{62}^{2} s_{72}^{2}\right.\right.} \\
& \left.\left.-s_{42} s_{52} s_{72}^{3}-s_{32} s_{62} s_{72}^{3}+s_{22} s_{72}^{4}-3 s_{42}^{2} s_{62} s_{72} s_{82}+2 s_{32} s_{42} s_{72}^{2} s_{82}+2 s_{42}^{3} s_{82}^{2}\right): s_{7}\right] .
\end{aligned}
$$

Here, the first section $S_{0}^{Z}$ is the point at infinity, while the second section $S_{1}^{Z}$ can be seen also in the affine chart. We note that $S_{0}^{Z}$ can be obtained by a simple coordinate transformation $^{7}$ from $S_{0}$ defined in (4.3), while $S_{1}^{Z}$ needs to be constructed using the procedure of Deligne applied in [22].

\subsubsection{Structure group $\mathrm{U}(1) \times \mathrm{U}(1): \mathrm{E}_{7} \times \mathrm{E}_{\mathbf{7}} \times \mathrm{U}(\mathbf{1})$ gauge symmetry}

We start with a model which has a heterotic vector bundle of structure group $\mathrm{U}(1) \times \mathrm{U}(1)$. Upon commutation within the group $\mathrm{E}_{8} \times \mathrm{E}_{8}$, the centralizer is given as $\mathrm{E}_{7} \times \mathrm{U}(1) \times \mathrm{E}_{7} \times$ $\mathrm{U}(1)$. On the heterotic side, the two $\mathrm{U}(1)$ factors acquire a mass term so that only a linear combination of them is massless. This matches the F-theory gauge group given by $\mathrm{E}_{7} \times \mathrm{E}_{7} \times \mathrm{U}(1)$.

Our example is specified by the following non-vanishing coefficients:

\begin{tabular}{|c|c|c|c|}
\hline Coefficient & $X$ & $X^{-}$ & $X^{+}$ \\
\hline$s_{1}$ & $s_{11} U^{2}+s_{12} U V+s_{13} V^{2}$ & $s_{12} U+s_{13} \mu$ & $s_{11} \lambda_{1}+s_{12} V$ \\
$s_{2}$ & $s_{22} U V$ & $s_{22} U$ & $s_{22} V$ \\
$s_{3}$ & $s_{32} U V$ & $s_{32} U$ & $s_{32} V$ \\
$s_{4}$ & $s_{42} U V$ & $s_{42} U$ & $s_{42} V$ \\
$s_{5}$ & $s_{52} U V$ & $s_{52} U$ & $s_{52} V$ \\
$s_{6}$ & $s_{62} U V$ & $s_{62} U$ & $s_{62} V$ \\
$s_{7}$ & $s_{72} U V$ & $s_{72} U$ & $s_{72} V$ \\
$s_{8}$ & $s_{82} U V$ & $s_{82} U$ & $s_{82} V$ \\
\hline
\end{tabular}

Here, the first columns denotes the coefficient in the Calabi-Yau constraint (3.2), the second column indices the chosen specialization and the third as well as fourth column contain the resulting coefficient in the half $\mathrm{K} 3$ fibrations $X^{ \pm}$, respectively.

Using the identities (3.16) and (3.21), we readily write down the defining equations for the half K3 surfaces $X_{2}^{ \pm}$obtained via stable degeneration explicitly. They read

$$
\begin{array}{cc}
X^{+}: & \left(s_{11} \lambda_{1}+s_{12} V\right) x_{1}^{4}+s_{22} V x_{1}^{3} x_{2}+s_{32} V x_{1}^{2} x_{2}^{2}+s_{42} V x_{1} x_{2}^{3} \\
& +s_{52} V x_{1}^{2} x_{3}+s_{62} V x_{1} x_{2} x_{3}+s_{72} V x_{2}^{2} x_{3}+s_{82} V x_{3}^{2}=0, \\
X^{-}: \quad\left(s_{12} U\right. & \left.+s_{13} \mu\right) x_{1}^{4}+s_{22} U x_{1}^{3} x_{2}+s_{32} U x_{1}^{2} x_{2}^{2}+s_{42} U x_{1} x_{2}^{3} \\
+ & +s_{52} U x_{1}^{2} x_{3}+s_{62} U x_{1} x_{2} x_{3}+s_{72} U x_{2}^{2} x_{3}+s_{82} U x_{3}^{2}=0 .
\end{array}
$$

\footnotetext{
${ }^{7}$ To be more precise, we refer in this case to (4.3) as a section of the heterotic geometry.
} 


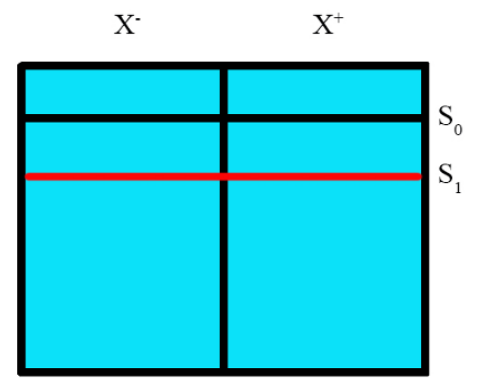

Figure 6. This figure shows the stable degeneration limit of a $K 3$ surface which has $E_{7} \times E_{7} \times U(1)$ gauge symmetry. There are the two half $\mathrm{K} 3$ surfaces, $X^{+}$and $X^{-}$which have both an $\mathrm{E}_{7}$ singularity and intersect in a common elliptic curve. Both have two sections, $S_{0}$ and $S_{1}$ which meet in the common elliptic curve. Thus, there are two global sections in the full K3 surface and therefore a $\mathrm{U}(1)$ factor.

By explicitly evaluating the Tate coefficients (A.3), one obtains the following orders of vanishing for the Tate vector at the loci $U=0$ and $V=0$ for the full K3 surface,

$$
\vec{t}_{U}=\vec{t}_{V}=(1,2,3,3,5,9),
$$

which reveal two $\mathrm{E}_{7}$ singularities. Also, the two half $\mathrm{K} 3$ surfaces inherit an $\mathrm{E}_{7}$ singularity each, which are located at $U=0, V=0$, respectively. Thus, the non-Abelian part of the gauge group is given by $\mathrm{E}_{7} \times \mathrm{E}_{7}$. Both half $\mathrm{K} 3$ surfaces have two rational sections given by $S_{0}^{X^{ \pm}}=[0: 1: 0]$ and $S_{1}^{X^{+}}=\left[0: s_{82} V:-s_{72} s_{82} V^{2}\right]$ and $S_{1}^{X^{-}}=\left[0: s_{82} U:-s_{72} s_{82} U^{2}\right]$, respectively. In the intersection point of the two half K3's given by $\left[U: \lambda_{1}: \mu: V: \lambda_{2}\right]=$ $[1: 0: 0: 1: 1]$, the sections $S_{0}^{X^{ \pm}}$from both half K3's intersect and meet each other, and similarly the sections $S_{1}^{X^{ \pm}}$from both half K3's intersect and meet each other, cf. figure 6 . Thus, the six-dimensional gauge group contains a $\mathrm{U}(1)$ factor.

However, if one evaluates the spectral cover, as described in section 4.1.2, one obtains ${ }^{8}$

$$
p_{+}=s_{11} x_{1}^{4}, \quad p_{-}=s_{13} x_{1}^{4} .
$$

which is mapped by use of the transformations (A.9) onto

$$
p_{+}^{W}=s_{11} z^{4}, \quad p_{-}^{W}=s_{13} z^{4} .
$$

These expressions gives rise to a constant spectral cover in affine Weierstrass coordinates $x, y$ defined by $z=1$. However, on an elliptic curve there does not exist any function which has exactly one zero at a single point, in this case $S_{1}{ }^{9}$ Nevertheless, one can use the two points $S_{0}^{Z}$ and $S_{1}^{Z}$ on the heterotic elliptic curve in order to construct the bundle $\mathcal{L}=\mathcal{O}\left(S_{1}^{Z}-S_{0}^{Z}\right)$ fiberwise, which is symmetrically embedded into both $\mathrm{E}_{8}$-bundles. As argued in [49], this bundle promotes to a bundle $\mathcal{L}^{6 D}$ in $6 \mathrm{D}$ whose first Chern class is

\footnotetext{
${ }^{8}$ Here, and in the following we set $x_{4} \rightarrow 1, x_{5} \rightarrow 1$ for convenience.

${ }^{9}$ However, note that the homogeneous expression $x_{1}^{4}$ vanishes at the loci of $S_{0}^{Z}$ and of $S_{1}^{Z}$. Indeed, the common solutions of $\lim _{x_{4} \rightarrow 1} \lim _{x_{5} \rightarrow 1} \chi$ defined in (4.2) with $x_{1}=0$ are given by $\left[x_{1}: x_{2}: x_{3}\right]=[0: 1: 0]$ or $\left[0: s_{8}:-s_{7} s_{8}\right]$.
} 
given by the difference of the two sections $c_{1}\left(\mathcal{L}^{6 D}\right)=\sigma_{S_{1}^{Z}}-\sigma_{S_{0}^{Z}}$, up to fiber contributions. Thus, the heterotic gauge group is given by $\mathrm{E}_{7} \times \mathrm{E}_{7} \times \mathrm{U}(1) \times \mathrm{U}(1)$. Due to the background bundle $\mathcal{L}^{6 D}$, these two U(1)'s seem both massive according to the Stückelberg mechanism discussed in section 2.4. However, due to the symmetric embedding into both $\mathrm{E}_{8}$ 's their sum remains massless. Thus, one obtains a perfect match with the F-theory gauge group.

We conclude with the remark that one can interpret this model also as a Higgsing of a model with $\mathrm{E}_{8} \times \mathrm{E}_{8}$ gauge symmetry as presented in the appendix B.1. Here, the Higgsing corresponds to a geometrical transition from the ambient space geometry $\mathbb{P}^{(1: 2: 3)}$ to $\mathrm{Bl}_{1} \mathbb{P}^{(1,1,2)}$ where the vacuum expectation value of the Higgs corresponds to the nonvanishing coefficient $s_{72}$.

\subsubsection{Structure group $\mathrm{U}(1) \times \mathrm{S}(\mathrm{U}(2) \times \mathrm{U}(1)): \mathrm{E}_{\mathbf{7}} \times \mathrm{E}_{6} \times \mathrm{U}(\mathbf{1})$ gauge symmetry}

As a next step, we investigate an example which has $\mathrm{E}_{7} \times \mathrm{E}_{6} \times \mathrm{U}(1)$ gauge symmetry. On the heterotic side we find an $\mathrm{U}(1) \times \mathrm{S}(\mathrm{U}(2) \times \mathrm{U}(1))$ structure group which directly matches the non-Abelian gauge group and gives rise to one massless as well as one massive $\mathrm{U}(1)$. The model is specified by the following non-vanishing coefficients:

\begin{tabular}{|c|c|c|c|}
\hline Coefficient & $X$ & $X^{-}$ & $X^{+}$ \\
\hline$s_{1}$ & $s_{11} U^{2}+s_{12} U V+s_{13} V^{2}$ & $s_{12} U+s_{13} \mu$ & $s_{11} \lambda_{1}+s_{12} V$ \\
$s_{2}$ & $s_{21} U^{2}+s_{22} U V$ & $s_{22} U$ & $s_{21} \lambda_{1}+s_{22} V$ \\
$s_{3}$ & $s_{32} U V$ & $s_{32} U$ & $s_{32} V$ \\
$s_{4}$ & $s_{42} U V$ & $s_{42} U$ & $s_{42} V$ \\
$s_{5}$ & $s_{52} U V$ & $s_{52} U$ & $s_{52} V$ \\
$s_{6}$ & $s_{62} U V$ & $s_{62} U$ & $s_{62} V$ \\
$s_{7}$ & $s_{72} U V$ & $s_{72} U$ & $s_{72} V$ \\
$s_{8}$ & $s_{82} U V$ & $s_{82} U$ & $s_{82} V$ \\
\hline
\end{tabular}

The evaluation of the order of vanishing of the Tate coefficients is summarized in the two Tate vectors

$$
\vec{t}_{V}=(1,2,2,3,5,8) \quad \vec{t}_{U}=(1,2,3,3,5,9) .
$$

It signal one $\mathrm{E}_{6}$ singularity at $V=0$ and one $\mathrm{E}_{7}$ singularity at $U=0$. The $\mathrm{E}_{7}$ singularity is inherited by the half $\mathrm{K} 3$ surface $X^{-}$while the $\mathrm{E}_{6}$ singularity is contained in the half $\mathrm{K} 3$ surface $X^{+}$after stable degeneration.

Next, we turn to the heterotic side. Here, the analysis of sections and U(1) symmetries from the perspective of the gluing condition is completely analogous to the geometry with $\mathrm{E}_{7} \times \mathrm{E}_{7} \times \mathrm{U}(1)$ gauge symmetry discussed in the previous section 4.2.1. The situation at hand is summarized in figure 7. However, there is a crucial difference in the evaluation of the spectral cover which we discuss next.

The corresponding split of the two half K3 surfaces into a spectral cover polynomial and the heterotic elliptic curve results in

$$
p^{+}=s_{11} x_{1}^{4}+s_{21} x_{1}^{3} x_{2}, \quad p^{-}=s_{13} x_{1}^{4} .
$$

Again, in order to evaluate the spectral cover information, one needs to transform both constraints into Weierstrass normal form. $p^{-}$is again just a constant and its interpretation 


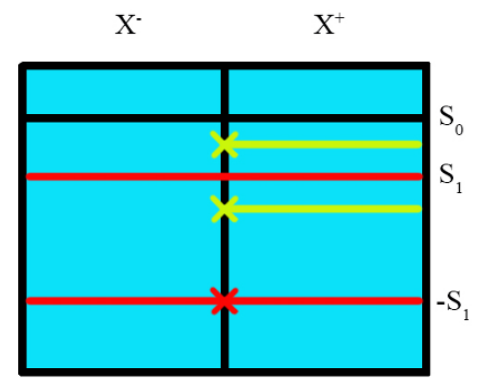

Figure 7. The interpretation of this figure is similar to figure 6 . The additional structure arises from two sections shown in yellow which form together with $\boxminus S_{1}$ the zeros of the spectral cover.

is along the lines of the previous example in section 4.2.1. However, in the case of $p^{+}$ something non-trivial happens. Its transform into Weierstrass coordinates reads explicitly

$$
\begin{aligned}
p_{W}^{+}= & s_{21} s_{62}^{3} s_{72}-4 s_{21} s_{52} s_{62} s_{72}^{2}-2 s_{11} s_{62}^{2} s_{72}^{2}+8 s_{11} s_{52} s_{72}^{3}-2 s_{21} s_{42} s_{62}^{2} s_{82} \\
& -4 s_{21} s_{42} s_{52} s_{72} s_{82}-4 s_{21} s_{32} s_{62} s_{72} s_{82}+24 s_{11} s_{42} s_{62} s_{72} s_{82}+12 s_{21} s_{22} s_{72}^{2} s_{82} \\
& -16 s_{11} s_{32} s_{72}^{2} s_{82}+8 s_{21} s_{32} s_{42} s_{82}^{2}-24 s_{11} s_{42}^{2} s_{82}^{2}-12 s_{21} s_{62} s_{72} x \\
& \left.+24 s_{11} s_{72}^{2} x+24 s_{21} s_{42} s_{82} x+24 s_{21} s_{72} y\right) /\left(2 \left(-s_{62}^{2} s_{72}^{2}+4 s_{52} s_{72}^{3}\right.\right. \\
& \left.\left.+12 s_{42} s_{62} s_{72} s_{82}-8 s_{32} s_{72}^{2} s_{82}-12 s_{42}^{2} s_{82}^{2}+12 s_{72}^{2} x\right)\right) .
\end{aligned}
$$

In contrast to the well-known case of the spectral cover in the $\mathbb{P}^{(1,2,3)}$-model which takes only poles at infinity, one observes that the denominator of (4.22) has two zeros at $S_{1}^{Z}$ and at $\boxminus S_{1}^{Z}$, the negative of $S_{1}^{Z}$ in the Mordell-Weil group of $Z$. In addition, the numerator has zeros at two irrational points $Q_{1}, Q_{2}$ and at $\boxminus S_{1}$. Finally, there is a pole of order one at $S_{0}^{Z}$. Here, $S_{0}^{Z}$ and $S_{1}^{Z}$ refer to the two sections (4.14), (4.15). Thus, the divisor of $p_{W}^{+}$is given by

$$
\operatorname{div}\left(p_{W}^{+}\right)=Q_{1}+Q_{2}-S_{1}-S_{0} .
$$

Clearly, in order to promote the points defined by the spectral cover polynomial in $8 \mathrm{D}$ to a curve in $6 \mathrm{D}$, the current form of $p_{W}^{-}$is not suitable due to its non-trivial denominator. However, one observes that the polynomial given by the numerator of $p_{W}^{+}$gives rise to the divisor

$$
\operatorname{div}\left(\operatorname{Numerator}\left(p_{W}^{+}\right)\right)=Q_{1}+Q_{2}+\boxminus S_{1}-3 S_{0}
$$

which is, however, linearly equivalent ${ }^{10}$ to the divisor (4.23). Consequently, a spectral cover, valid also for the construction of lower-dimensional compactifications, is defined by the numerator of (4.22).

Thus, the three zeros $Q_{1}, Q_{2}$ and $\boxminus S_{1}$ form, following section 2.2, a split $\mathrm{SU}(3)$ spectral cover, i.e. an $\mathrm{S}(\mathrm{U}(2) \times \mathrm{U}(1))$ spectral cover. All three points extend as sections into the half K3 surface $X^{+}$, cf. figure 7 . Two of these sections are linearly independent and are in

\footnotetext{
${ }^{10}$ To see this, one notices that the element $-S_{1}+S_{0}$ in $\operatorname{Pic}^{0}(E)$ is equivalent to $-S_{1}+S_{0}+f$ where $f$ is defined as $x-x_{S_{1}}$ on $E$ with $x_{S_{1}}$ denoting the $x$-coordinate of $S_{1}$. It holds that $\operatorname{div}(f)=S_{1}+\boxminus S_{1}-2 S_{0}$. Thus, $-S_{1}+S_{0}$ maps to $\boxminus S_{1}$ on $E$ under the map (2.11).
} 
8D the generators of the rank two Mordell-Weil group corresponding to a rational elliptic surface with an $\mathrm{E}_{6}$ singularity. However, due to monodromies of $Q_{1}$ and $Q_{2}$ only $\boxminus S_{1}$ survives in $6 \mathrm{D}$ as a rational section.

In conclusion, this spectral cover gives rise to an $\mathrm{S}(\mathrm{U}(2) \times \mathrm{U}(1))$ background bundle which is embedded into the $\mathrm{E}_{8}$ factor corresponding to $X^{+}$. The centralizer of this is given by $\mathrm{E}_{6} \times \mathrm{U}(1)$. The latter factor seems again massive due to the $\mathrm{U}(1)$ background bundle. However, this $\mathrm{U}^{(1)}$ forms together with the seemingly massive $\mathrm{U}(1)$ of the half K3 surface $X^{-}$a massless linear combination. In conclusion, there is a perfect match with the F-theory analysis of the low energy gauge group. Analogously to the previous case in section 4.2.1, this model can be understood as arising by Higgsing the non-Abelian model B.2 with gauge symmetry $E_{8} \times E_{7}$. Here, a (massive) $U(1)$ factor is embedded minimally into both factors. Again, the vacuum expectation value of the Higgs corresponds to the coefficient $s_{72}$. In addition, we can view this model also as arising by a Higgsing process from a compactification with $\mathrm{E}_{7} \times \mathrm{E}_{7} \times \mathrm{U}(1)$ gauge group where a vacuum expectation value of the Higgs corresponds to $s_{21}$.

\subsubsection{Structure group $\mathrm{U}(1) \times(\mathrm{SU}(2) \times \mathrm{SU}(2) \times \mathrm{U}(1)): \mathrm{E}_{7} \times \mathrm{SO}(9) \times \mathrm{U}(1)$ gauge symmetry}

The final example in this chain of Higgsings is given by a model with $\mathrm{E}_{7} \times \mathrm{SO}(9) \times \mathrm{U}(1)$ gauge symmetry. On the heterotic side we find an $\mathrm{U}(1) \times(\mathrm{SU}(2) \times \mathrm{SU}(2) \times \mathrm{U}(1))$ structure group which matches the non-Abelian gauge content. Also in this case we find one massless as well as one massive $\mathrm{U}(1)$ on the heterotic side.

As before, we define the model by the following choice of coefficients in $X$ :

\begin{tabular}{|c|c|c|c|}
\hline Coefficient & $X$ & $X^{-}$ & $X^{+}$ \\
\hline$s_{1}$ & $s_{11} U^{2}+s_{12} U V+s_{13} V^{2}$ & $s_{12} U+s_{13} \mu$ & $s_{11} \lambda_{1}+s_{12} V$ \\
$s_{2}$ & $s_{21} U^{2}+s_{22} U V$ & $s_{22} U$ & $s_{21} \lambda_{1}+s_{22} V$ \\
$s_{3}$ & $s_{31} U^{2}+s_{32} U V$ & $s_{32} U$ & $s_{31} \lambda_{1}+s_{32} V$ \\
$s_{4}$ & $s_{42} U V$ & $s_{42} U$ & $s_{42} V$ \\
$s_{5}$ & $s_{52} U V$ & $s_{52} U$ & $s_{52} V$ \\
$s_{6}$ & $s_{62} U V$ & $s_{62} U$ & $s_{62} V$ \\
$s_{7}$ & $s_{72} U V$ & $s_{72} U$ & $s_{72} V$ \\
$s_{8}$ & $s_{82} U V$ & $s_{82} U$ & $s_{82} V$ \\
\hline
\end{tabular}

Once again we begin the analysis on the F-theory side with the evaluation of the order of vanishing of the Tate coefficients. We obtain the Tate vectors

$$
\vec{t}_{V}=(1,1,2,3,4,7), \quad \vec{t}_{U}=(1,2,3,3,5,9),
$$

which signal one $\mathrm{SO}(9)$ singularity at $V=0$ and one $\mathrm{E}_{7}$ singularity at $U=0$, each of which being inherited by one half K3 surface.

For the analysis of the heterotic side, we split the two half K3 surfaces into a spectral cover polynomial and the heterotic elliptic curve. We obtain

$$
p^{+}=s_{11} x_{1}^{4}+s_{21} x_{1}^{3} x_{2}+s_{31} x_{1}^{2} x_{2}^{2}, \quad p^{-}=s_{11} x_{1}^{4},
$$




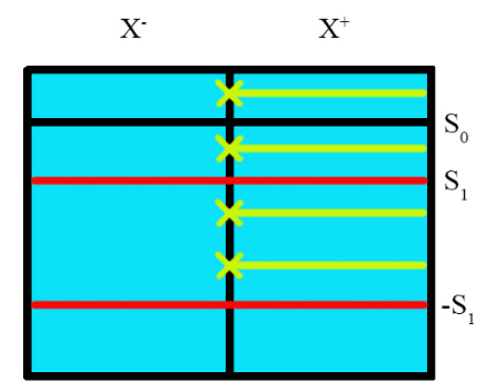

Figure 8. The half K3 surface $X^{-}$only exhibits the section $S^{1}$ in addition to the zero section. In contrast, $X^{+}$gives rise to a spectral cover polynomial that has two pairs of irrational solutions $Q_{1}$, $Q_{2}, R_{1}, R_{2}$ that sum up to $S_{1}^{Z}$ each.

from which we see that $p^{-}$is again a trivial spectral cover. Again, in order to evaluate the non-trivial spectral cover $p^{+}$, one needs to transform both constraints into Weierstrass normal form. The interpretation of $p^{+}$is as in the previous cases. We again obtain a Weierstrass form $p_{W}^{+}$with a denominator. The explicit expression is rather lengthy and can be provided upon request. Its divisor is given by

$$
\operatorname{div}\left(p_{W}^{+}\right)=Q_{1}+Q_{2}+R_{1}+R_{2}-2 S_{1}-2 S_{0}
$$

Here $Q_{1}, Q_{2}$ and $R_{1}, R_{2}$ are two pairs of irrational points which obey $Q_{1} \boxplus Q_{2} \boxminus S_{1}=0$ and $R_{1} \boxplus R_{2} \boxminus S_{1}=0$. The divisor of $p_{W}^{+}$is again equivalent to the divisor of its numerator reading

$$
\operatorname{div}\left(\operatorname{Numerator}\left(p_{W}^{+}\right)\right)=Q_{1}+Q_{2}+R_{1}+R_{2}+2 \boxminus S_{1}-6 S_{0} .
$$

By a similar token as before, we thus drop the denominator and just work with the numerator of $p_{W}^{+}$.

All the points appearing here extend to sections of the half $\mathrm{K} 3$ surface $X^{+}$. However, while $Q_{1}, Q_{2}, R_{1}, R_{2}$ extend to rational sections of the half $\mathrm{K} 3$ surface they do not lift to rational sections of the rational elliptic threefold. Altogether, we obtain as in the previous examples two rational sections in both half $\mathrm{K} 3$ surfaces which glue to global sections and therefore give rise to a $\mathrm{U}(1)$ factor. Besides that the spectral cover is split and describes a vector bundle with structure group $\mathrm{S}(\mathrm{U}(2) \times \mathrm{U}(1)) \times \mathrm{S}(\mathrm{U}(2) \times \mathrm{U}(1))$, where the $\mathrm{U}(1)$ part in both factors needs to be identified. This is due to the fact that in both cases the same point, $\boxminus S_{1}^{Z}$, splits off. Thus, the spectral cover is isomorphic to $\mathrm{SU}(2) \times \mathrm{SU}(2) \times \mathrm{U}(1)$ whose centralizer ${ }^{11}$ within $\mathrm{E}_{8}$ is given by $\mathrm{SO}(9) \times \mathrm{U}(1)$. Thus, we obtain again two seemingly massive U(1)'s which give rise to one massless linear combination.

This model can be understood by a Higgsing mechanism. Either it can be viewed as arising from the non-Abelian model in section B.3 with $\mathrm{E}_{8} \times \mathrm{SO}(11)$ gauge symmetry, by giving a vacuum expectation value to a Higgs corresponding to $s_{72}$, or from the previous example in section 4.2 .2 , by giving a vacuum expectation value to a Higgs associated to $s_{31}$.

\footnotetext{
${ }^{11}$ We employ here the breaking $\mathrm{E}_{8} \longrightarrow \mathrm{SO}(9) \times \mathrm{SU}(2) \times \mathrm{SU}(2) \times \mathrm{SU}(2)$.
} 


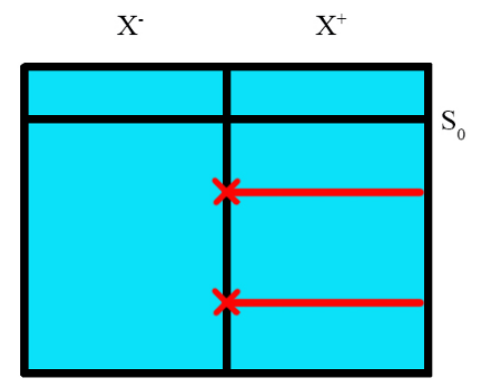

Figure 9. The half K3 surface $X^{-}$has only one section $S_{0}^{X^{-}}$which merges with the section $S_{0}^{X^{+}}$ from the other half $\mathrm{K} 3$ surface $X^{+} . X^{+}$has in addition also the section $S^{X^{+}}$which does not merge with a section of $X^{-}$. Thus, there is no $\mathrm{U}(1)$-factor on the F-theory side.

\subsubsection{Example with only one massive $\mathrm{U}(1): \mathrm{S}(\mathrm{U}(1) \times \mathrm{U}(1))$ structure group}

Finally, we conclude the list of examples with a model which has only one U(1)-bundle embedded into one of its $\mathrm{E}_{8}$ factors while the other $\mathrm{E}_{8}$ stays untouched. Accordingly there is only one massive $\mathrm{U}(1)$ symmetry. On the F-theory side we obtain an $\mathrm{E}_{8} \times \mathrm{E}_{6} \times \mathrm{SU}(2)$ gauge symmetry which matches the findings on the heterotic side.

The model is defined by the following specialization of the coefficients in the constraint (4.2):

\begin{tabular}{|c|c|c|c|}
\hline Coefficient & $X$ & $X^{-}$ & $X^{+}$ \\
\hline$s_{1}$ & $s_{13} V^{2}$ & $s_{13} \mu$ & 0 \\
$s_{2}$ & $s_{22} U V$ & $s_{22} U$ & $s_{22} V$ \\
$s_{3}$ & $s_{32} U V$ & $s_{32} U$ & $s_{32} V$ \\
$s_{4}$ & $s_{41} U^{2}+s_{42} U V$ & $s_{42} U$ & $s_{42} V+s_{41} \lambda_{1}$ \\
$s_{5}$ & $s_{52} U V$ & $s_{52} U$ & $s_{52} V$ \\
$s_{6}$ & $s_{62} U V$ & $s_{62} U$ & $s_{62} V$ \\
$s_{7}$ & 0 & 0 & 0 \\
$s_{8}$ & $s_{82} U V$ & $s_{82} U$ & $s_{82} V$ \\
\hline
\end{tabular}

First of all, we note that the coefficient $s_{7}$ vanishes identically. Thus, we have changed the ambient space of the fiber from $\mathrm{Bl}_{1} \mathbb{P}^{(1,1,2)}$ to $\mathbb{P}^{(1,2,3)}$. Therefore, we do not expect to see another section besides the zero section on the F-theory side and therefore no U(1), cf. appendix C.

First, we determine the gauge group on the F-theory side. As before, we evaluate the Tate coefficients along the singular fibers which are in the case at hand located at $U=0$, $V=0$ and $s_{41} U+s_{42} V=0$. One obtains the Tate vectors

$$
\vec{t}_{U}=(1,2,3,4,5,10), \quad \vec{t}_{V}=(1,2,2,3,5,8), \quad \vec{t}_{s_{41} U+s_{42} V}=(0,0,1,1,2,2) .
$$

Clearly, these signal an $\mathrm{E}_{8} \times \mathrm{E}_{6} \times \mathrm{SU}(2)$ gauge group in F-theory. Also, after the stable degeneration limit, one obtains one half $\mathrm{K} 3$ surface $X^{-}$with an $\mathrm{E}_{8}$ singularity and one, $X^{+}$, with an $\mathrm{E}_{6} \times \mathrm{SU}(2)$ singularity.

For the further analysis we remark that there is the zero section $S_{0}=[0: 1: 0]$ in the K3 surface only. As in the previous discussion, we refer to the $\mathbb{P}^{(1,1,2)}$ coordinates 
$\left[x_{1}: x_{2}: x_{3}\right]$ only, i.e. we work in the limit $x_{4} \rightarrow 1, x_{5} \rightarrow 1$. For the two half K3s one finds that $X^{-}$has only a zero section. In contrast, one observes the sections ${ }^{12} S^{X^{+}}=[1: 0: 0]$ and $\boxminus S^{X^{+}}=\left[s_{82} V: 0:-s_{52} s_{82} V^{2}\right]$ in the other half K3 surface $X^{+}$. However, these sections do not glue with another section of $X^{-}$and therefore do not give rise to a $\mathrm{U}(1)$ symmetry from the F-theory perspective. However, from the heterotic perspective they should give rise to a massive $\mathrm{U}(1)$ which upon commutation within $\mathrm{E}_{8}$ leaves an $\mathrm{E}_{6} \times \mathrm{SU}(2)$ gauge symmetry.

This result is in agreement with the spectral cover analysis. One evaluates the spectral cover polynomials as

$$
p^{-}=s_{13} x_{1}^{4} \quad p^{+}=s_{41} x_{1} x_{2}^{3} .
$$

As observed already before, the Weierstrass transform $p_{W}^{-}$of $p^{-}$does not have any common solution with the heterotic elliptic curve and therefore the $\mathrm{E}_{8}$-symmetry does not get broken. For the half K3 surface $X^{+}$, the common solutions to $p_{W}^{+}$and the heterotic elliptic curve are given in Weierstrass coordinates $[x: y: z]$ as

$$
\begin{aligned}
S_{W}^{Z} & =\left[\frac{1}{12}\left(s_{62}^{2}-4 s_{32} s_{82}\right):-\frac{1}{2} s_{42} s_{52} s_{82}: 1\right], \\
\boxminus S_{W}^{Z} & =\left[\frac{1}{12}\left(s_{62}^{2}-4 s_{32} s_{82}\right): \frac{1}{2} s_{42} s_{52} s_{82}: 1\right] .
\end{aligned}
$$

Here, $S_{W}^{Z}$ and $\boxminus S_{W}^{Z}$ denote the intersections of $S^{X^{+}}$and $\boxminus S^{X^{+}}$with the heterotic geometry $Z$ respectively, in Weierstrass coordinates. Thus, we observe a split spectral cover pointing towards the structure group $S(\mathrm{U}(1) \times \mathrm{U}(1))$. Using the breaking $\mathrm{E}_{8} \longrightarrow \mathrm{E}_{6} \times \mathrm{SU}(2) \times \mathrm{U}(1)$, this spectral cover matches with the observed gauge group. The $\mathrm{U}(1)$ is decoupled from the massless spectrum via the Stückelberg effect of section 2.4.

\subsection{Split spectral covers with torsional points}

In the following, we discuss examples which exhibit a torsional section in their spectral covers. As mentioned before, heterotic/F-theory duality suggests that the structure group of the heterotic vector bundle should contain a discrete part.

\subsubsection{Structure group $\mathbb{Z}_{2}: \mathrm{E}_{8} \times \mathrm{E}_{7} \times \mathrm{SU}(2)$ gauge symmetry}

We consider a model which arises by the following specialization of coefficients in (4.2):

\begin{tabular}{|c|c|c|c|}
\hline Coefficient & $X$ & $X^{-}$ & $X^{+}$ \\
\hline$s_{1}$ & $s_{13} V^{2}$ & $s_{13} \mu$ & 0 \\
$s_{2}$ & $s_{22} U V$ & $s_{22} U$ & $s_{22} V$ \\
$s_{3}$ & $s_{32} U V$ & $s_{32} U$ & $s_{32} V$ \\
$s_{4}$ & $s_{41} U^{2}+s_{42} U V$ & $s_{42} U$ & $s_{42} V+s_{41} \lambda_{1}$ \\
$s_{5}$ & 0 & 0 & 0 \\
$s_{6}$ & $s_{62} U V$ & $s_{62} U$ & $s_{62} V$ \\
$s_{7}$ & 0 & 0 & 0 \\
$s_{8}$ & $s_{82} U V$ & $s_{82} U$ & $s_{82} V$ \\
\hline
\end{tabular}

\footnotetext{
${ }^{12}$ Clearly, as the rank of the Mordell Weil group of $X^{+}$is positive, there are in fact infinitely many sections.
} 


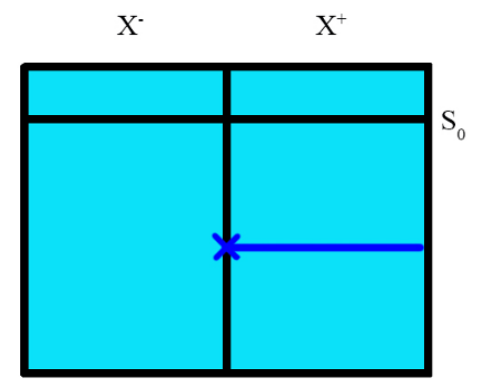

Figure 10. The stable degeneration limit of a $\mathrm{K} 3$ surface with $\mathrm{E}_{8} \times\left(\mathrm{E}_{7} \times \mathrm{SU}(2)\right) / \mathbb{Z}_{2}$. The half K3 surface $X^{-}$has trivial Mordell-Weil group, while the half K3 surface $X^{+}$has a torsional Mordell-Weil group $\mathbb{Z}_{2}$.
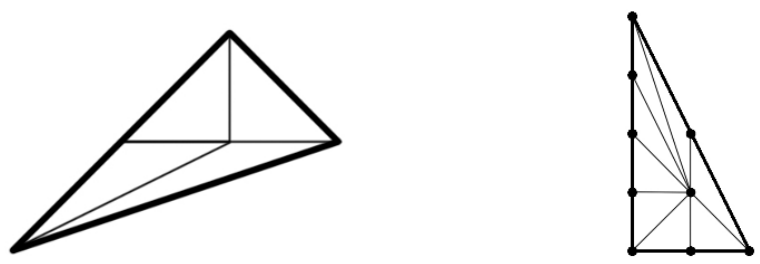

Figure 11. The left picture shows the specialized two-dimensional polytope $\Delta_{2}$ corresponding to the monomials parameterizing the elliptic fiber of the half K3 surface $X^{+}$. The right figure shows its dual, $\Delta_{2}^{\circ}$, which specifies the ambient space of the elliptic fiber of $X^{+}$.

We start the analysis with the gauge group on the F-theory side first. There are three singular loci of the fibration at $U=0, V=0$ and $s_{41} U+s_{42} V=0$. The evaluation of the Tate coefficients reveals the Tate vectors

$$
\vec{t}_{U}=(1,2, \infty, 4,5,10), \quad t_{V}=(1,2, \infty, 3,5,9), \quad \vec{t}_{s_{41} U+s_{42} V}=(0,0, \infty, 1,2,2) .
$$

Thus, there are an $\mathrm{E}_{8}$ singularity as well as an $\mathrm{E}_{7}$ and an $\mathrm{SU}(2)$ singularity. The $\mathrm{E}_{8}$ singularity is inherited by the half $\mathrm{K} 3$ surface $X^{-}$while $X^{+}$gets endowed with an $\mathrm{E}_{7}$ and an $\mathrm{SU}(2)$ singularity.

As a next step, we observe that there is only one section given by $[1: 0: 0]$ in the half K3 surface $X^{-}$and two sections given by $\left[x_{1}: x_{2}: x_{3}\right]=[0: 1: 0]$ and $\left[x_{1}: x_{2}: x_{3}\right]=$ $[1: 0: 0]$ in the half $\mathrm{K} 3$ surface $X^{+}$. Here, we work again in the limit $x_{4}=x_{5}=1$. In contrast, the full K3 surface has only one section namely the point at infinity. Moreover, a transformation into Weierstrass coordinates shows that the generic section $S_{1}^{X^{+}}$has specialized into a torsional section of order two as can be checked using the results of [37]. This is expected, as the centralizer of the gauge algebra ${ }^{13} \mathrm{E}_{7} \times \mathrm{SU}(2)$ within $\mathrm{E}_{8}$ is given by $\mathbb{Z}_{2}$, which is also expected from the general analysis of [56]. In contrast, the full K3 surface $X$ does not have a torsional section of order two [57].

\footnotetext{
${ }^{13}$ To be precise, $\mathrm{E}_{8}$ only contains the group $\left(\mathrm{E}_{7} \times \mathrm{SU}(2)\right) / \mathbb{Z}_{2}$ as a subgroup.
} 
Finally, we turn towards the analysis of the gauge group from the heterotic side. Here, the spectral cover is given by

$$
p^{-}=s_{13} x_{1}^{4}, \quad p^{+}=s_{41} x_{1} x_{2}^{3} .
$$

After transformation to Weierstrass normal coordinates $p_{W}^{-}$is given by a constant which has no common solution with the elliptic curve. In contrast, the transformed quantity $p_{W}^{+}$ gives rise to the point

$$
[x: y: z]=\left[\frac{1}{3}\left(\frac{s_{62}^{2}}{4}-s_{32} s_{82}\right): 0: 1\right] .
$$

which is a torsion point of order two. In other words we see that the spectral cover is just given by a torsional point.

In [37] it has been suggested that an F-theory compactification with a torsional section in an elliptically fibered Calabi-Yau manifold and its stable degeneration limit should be dual to pointlike instantons with discrete holonomy on the heterotic side. Due to the similarity to the considered example, we propose that the spectral cover $p_{W}^{+}$is to be interpreted as describing such a pointlike instanton with discrete holonomy. In addition, as pointed out above, the matching of gauge symmetry on both sides of the duality only works if the spectral cover $p_{W}^{+}$is interpreted in this way. It would be exciting to confirm this proposal further by a more detailed analysis of the spectral cover, computation of the heterotic tadpole, or an analysis of codimension two singularities in F-theory.

\subsubsection{Structure group $\mathrm{S}\left(\mathrm{U}(2) \times \mathbb{Z}_{2}\right): \mathrm{E}_{8} \times \mathrm{E}_{6} \times \mathrm{U}(1)$ gauge symmetry}

In this section we present another example whose spectral cover polynomial contains a torsional point and leads to an $\mathrm{E}_{8} \times \mathrm{E}_{6} \times \mathrm{U}(1)$ gauge symmetry. As one $\mathrm{E}_{8}$ factor is left intact, the $\mathrm{U}(1)$ factor needs to be embedded solely into one $\mathrm{E}_{8}$ bundle.

The starting point of our analysis is the following specialization of coefficients in (4.2):

\begin{tabular}{|c|c|c|c|}
\hline Coefficient & $X$ & $X^{-}$ & $X^{+}$ \\
\hline$s_{1}$ & $s_{13} V^{2}$ & $s_{13} \mu$ & 0 \\
$s_{2}$ & $s_{22} U V$ & $s_{22} U$ & $s_{22} V$ \\
$s_{3}$ & $s_{32} U V$ & $s_{32} U$ & $s_{32} V$ \\
$s_{4}$ & $s_{41} U^{2}+s_{42} U V$ & $s_{42} U$ & $s_{42} V+s_{41} \lambda_{1}$ \\
$s_{5}$ & 0 & 0 & 0 \\
$s_{6}$ & $s_{62} U V$ & $s_{62} U$ & $s_{62} V$ \\
$s_{7}$ & $s_{71} U^{2}$ & 0 & $s_{71} \lambda_{1}$ \\
$s_{8}$ & $s_{82} U V$ & $s_{82} U$ & $s_{82} V$ \\
\hline
\end{tabular}

As in the previous cases, we compute the orders of vanishing of the Tate coefficients in order to determine the gauge group on the F-theory side. The computed Tate vectors signal an $\mathrm{E}_{8}$ symmetry at $U=0$ and an $\mathrm{E}_{6}$ symmetry at $V=0$. As a next step, we investigate the rational sections of $X$. As the coefficient $s_{7}$ does not vanish for the full K3 surface, there are the two generic sections $S_{0}, S_{1}$ realized in this model. However, the half $\mathrm{K} 3$ surface $X^{-}$only has the zero section $S_{0}^{X^{-}}$. In contrast, the half K3 surface $X^{+}$ 


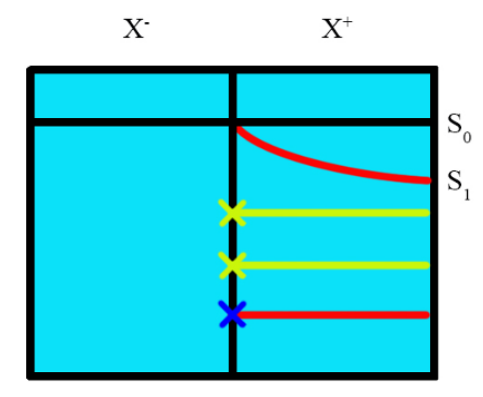

Figure 12. The half K3 surface $X^{-}$exhibits only the zero section, while the half K3 surface $X^{+}$ has also the section $S_{1}^{X^{+}}$which merges with the section $S_{0}^{X^{-}}$along the heterotic geometry. Thus there are two independent sections in the full $\mathrm{K} 3$ surface giving rise to a $\mathrm{U}(1)$ gauge group factor. In addition, the inverse of $S_{1}^{X^{+}}$becomes a torsion point of order two when hitting the heterotic geometry.

has two linear independent sections given by $S_{0}^{X^{+}}, S_{1}^{X^{+}}$, which lift to rational sections of the elliptic threefold. Due to the theory of rational elliptic surfaces, there is another linear independent section of $X^{+}$in $8 \mathrm{D}$ which nevertheless does not lift to a rational section of the rational elliptic threefold. The two linear independent rational sections unify in the heterotic elliptic curve and continue as one section into the other half K3 surface, see figure 12. This behavior of rational sections explains the origin of the $\mathrm{U}(1)$-factor from the gluing condition discussed in section 2.4.2.

As a further step, we investigate how this $U(1)$ factor is reflected in the spectral cover on the heterotic side. The spectral cover polynomials computed by stable degeneration read

$$
p^{-}=s_{13} x_{1}^{4}, \quad p^{+}=s_{41} x_{1} x_{2}^{3}+s_{71} x_{2}^{2} x_{3} .
$$

The interpretation of $p^{-}$is as in all the other cases just a trivial spectral cover. The common solution to $p^{+}$and the heterotic Calabi-Yau manifold $Z$ is given by a pair of irrational points $R_{1}, R_{2}$ as well as a further point $T_{t}$ which has in Weierstrass normal form coordinates

$$
T_{t}=\left[\frac{1}{3}\left(\frac{1}{4} s_{62}^{2}-s_{32} s_{82}\right): 0: 1\right] .
$$

Thus, it is a torsion point of order two. However, it does not extend as a full torsional section into the half $\mathrm{K} 3$ surface $X^{+}$. The corresponding section is rather the inverse of $S_{1}$.

Again we see that the split spectral cover $p^{+}$contains a torsional section. Let us comment on the interpretation of this for the structure group of the heterotic vector bundle. Heterotic/F-theory duality implies that the low-energy effective theory contains a massless $\mathrm{U}(1)$-symmetry. However, as we have seen in section 2.4, a $\mathrm{U}(1)$ background bundle in the heterotic theory has a non-trivial field strength and thus a non-vanishing first Chern class, which would yield a massive $\mathrm{U}(1)$ in the effective field theory. Thus, we can not interpret the torsional component $T_{t}$ to the spectral cover as a U(1) background bundle. By the arguments of section 2.2 and the similarity to the setups considered in [37], it is tempting to identify this torsional component $T_{t}$ as a pointlike heterotic instanton with discrete holonomy. In order to justify this statement, it would be necessary to compute 
the first Chern class of a heterotic line bundle that is defined in terms of components to the cameral cover given by rational sections of the half K3 fibrations arising in stable degeneration. In [49], it has been argued that the first Chern class is given, up to vertical components, by the difference of the rational section and the zero section. If the first Chern class would be completed into the Shioda map of the rational section, which we conjecture to be the case, it would be zero precisely for a torsional section [58]. Consequently, the $\mathrm{U}(1)$ in the commutant of $\mathrm{E}_{8}$ would remain massless as the gauging in (2.23) would be absent. It would be exciting to confirm this conjecture by working out the missing vertical part in the formula for the first Chern class of a $U(1)$ vector bundle.

\subsection{U(1) factors arising from purely non-Abelian structure groups}

In this final section, we present an example in which the heterotic vector bundle has only purely non-Abelian structure group, while the F-theory gauge group analysis clearly signals a $\mathrm{U}(1)$ factor.

As in the previous cases, we start by specifying the specialization of the coefficients in the defining hypersurface equation for $X$ :

\begin{tabular}{|c|c|c|c|}
\hline Coefficient & $X$ & $X^{-}$ & $X^{+}$ \\
\hline$s_{1}$ & $s_{12} U V+s_{13} V^{2}$ & $s_{12} U+s_{13} \mu$ & $s_{12} V$ \\
$s_{2}$ & $s_{22} U V$ & $s_{22} U$ & $s_{22} V$ \\
$s_{3}$ & $s_{32} U V$ & $s_{32} U$ & $s_{32} V$ \\
$s_{4}$ & $s_{42} U V$ & $s_{42} U$ & $s_{42} V$ \\
$s_{5}$ & $s_{52} U V$ & $s_{52} U$ & $s_{52} V$ \\
$s_{6}$ & $s_{62} U V$ & $s_{62} U$ & $s_{62} V$ \\
$s_{7}$ & $s_{71} U^{2}$ & 0 & $s_{71} \lambda_{1}$ \\
$s_{8}$ & $s_{82} U V$ & $s_{82} U$ & $s_{82} V$ \\
\hline
\end{tabular}

We determine the gauge symmetry of the F-theory side by analysis of the Tate coefficients. We obtain the Tate vectors

$$
\vec{t}_{U}=(1,2,3,4,5,10) \quad \vec{t}_{V}=(1,1,2,2,4,6),
$$

which reveal an $\mathrm{E}_{8}$ singularity at $U=0$ and an $\mathrm{SO}(7)$ singularity at $V=0$. We note that it is not directly possible to distinguish an $\mathrm{SO}(7)$ singularity from an $\mathrm{SO}(8)$ singularity using the Tate table 2 only. To confirm that the type of singularity is indeed $\mathrm{SO}(7)$ we have to investigate the monodromy cover [59] which is for an $I_{0}^{*}$ fiber given by

$$
A: \quad \psi^{3}+\left(\left.\frac{f}{v^{2}}\right|_{v=0}\right) \psi+\left(\left.\frac{g}{v^{3}}\right|_{v=0}\right)=0 .
$$

Here, $v$ is the affine coordinate $V / U$ and $f, g$ are the Weierstrass coefficients. An $I_{0}^{*}$ fiber is $\mathrm{SO}(7)$ if the monodromy cover $A$ factors into a quadratic and a linear constraint, which is indeed the case for the example at hand.

The stable degeneration limit yields two half K3 surfaces, $X^{+}$and $X^{-}$, cf. figure 13. There only exists the zero section in $X^{-}$. In contrast, $X^{+}$has two independent sections which are given by $S_{0}^{X^{+}}$and $S_{1}^{X^{+}}$and lift to rational sections of the rational elliptic 


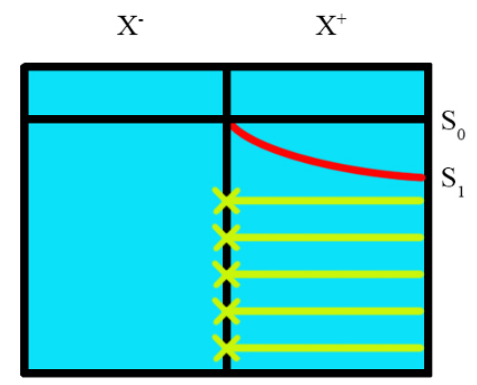

Figure 13. The half K3 surface $X^{-}$exhibits only the zero section, while the half K3 surface $X^{+}$ has also the section $S_{1}^{X^{+}}$which merges with the section $S_{0}^{X^{-}}$in the heterotic geometry. Thus, there are two independent sections in the full K3 surface giving rise to a U(1) gauge group factor.

threefold. ${ }^{14}$ As in the previously considered case in section $4.3 .2, S_{1}^{X^{+}}$unifies with $S_{0}^{X^{-}}$on the heterotic elliptic curve. Thus, there are two global sections in the full K3 surface and therefore a $\mathrm{U}(1)$ factor in the F-theory compactification.

Turning towards the discussion of the heterotic gauge bundles, one finds that the $\mathrm{U}(1)$ factor is encoded in the data of the spectral cover polynomial as follows. We observe that the spectral covers following $X^{+}$and $X^{-}$, respectively, are given by

$$
p^{-}=s_{13} x_{1}^{4}, \quad p^{+}=s_{71} x_{2}^{2} x_{3} .
$$

The intersection of its Weierstrass transform $p_{W}^{+}$with the heterotic elliptic curve gives five irrational points $R_{1}, R_{2}, T_{1}, T_{2}, T_{3}$ with $R_{1} \oplus R_{2}=0$ and $T_{1} \oplus T_{2} \oplus T_{3}=0$. Thus we have a heterotic vector bundle with $\mathrm{SU}(2) \times \mathrm{SU}(3)$ structure group. As the spectral cover $p^{+}$ has one free parameter only, namely $s_{71}$, this model does not seem to have any moduli.

As our understanding of the precise embedding of the structure group into $\mathrm{E}_{8}$ is limited, we have checked all possible ways to embed the group $\mathrm{SO}(7) \times \mathrm{SU}(2) \times \mathrm{SU}(3)$ into $\mathrm{E}_{8}$. Independently of the chosen embedding, there is always a $\mathrm{U}(1)$ in all possible breakings. Thus, we are led to conclude that the centralizer of $\mathrm{SU}(2) \times \mathrm{SU}(3)$ necessarily produces a $\mathrm{U}(1)$ factor which matches with the F-theoric analysis.

\section{Conclusions and future directions}

In this paper we have presented a first explicit analysis of the origin of Abelian gauge symmetries for string theory compactifications within the duality between the $\mathrm{E}_{8} \times \mathrm{E}_{8}$ heterotic string and F-theory. Here we summarize the framework of the analysis, highlight the key advancements, and conclude with future directions.

Framework. We have focused on F-theory compactifcations with a rank one MordellWeil group of rational sections both for compactifications to 8D and 6D. We have systematically studied a broad class F-theory compactifications on elliptically fibered Calabi-Yau $(n+1)$-folds (with $n=1,2$, respectively) with rational sections and rigorously performed the

\footnotetext{
${ }^{14}$ On the level of the rational elliptic surface $X_{2}^{+}$, there are additional linear independent sections which however do not lift into the six-dimensional geometry.
} 
stable degeneration limit to dual heterotic compactifications on elliptically fibered CalabiYau $n$-folds. All considered examples are toric hypersurfaces and the stable degeneration limit is performed as a toric symplectic cut.

The key aspects of the analysis are the following:

- We have carefully investigated the solutions of the spectral cover polynomial and the hypersurface for the heterotic elliptically fibered Calabi-Yau manifold. We have used the group law of the elliptic curve in Weierstrass normal form in order to determine the structure group of the heterotic background bundle.

- We have analyzed the origin of the resulting gauge group. In $6 \mathrm{D}$ this involves incorporation of the massive U(1) gauge symmetries, due to the heterotic Stückelberg mechanism, that are not visible in F-theory.

Key results. While the F-theory side provides a unifying treatment of Abelian gauge symmetries, as encoded in the Mordell-Weil group of elliptically fibered Calabi-Yau $(n+1)$ folds, a detailed analysis of a broad classes of toric F-theory compactifications has resulted in the proposal of three different classes of heterotic duals that give rise to $\mathrm{U}(1)$ gauge group factors:

- Split spectral covers describing bundles with $\mathrm{S}(\mathrm{U}(m) \times \mathrm{U}(1))$ structure group. Examples of this type have been discussed in section 4.2.

- Spectral covers containing torsional sections giving rise to bundles with $\mathrm{SU}(m) \times \mathbb{Z}_{k}$ structure group. Classes of examples with this structure group have been presented in section 4.3.

- The appearance of bundles with structure groups of the type $\mathrm{SU}(m) \times \mathrm{SU}(l)$ whose commutants inside $\mathrm{E}_{8}$ contain a $\mathrm{U}(1)$-factor. Explicit examples of this form can be found in section 4.4 .

Future directions. While the work presents a pioneering effort, addressing comprehensively the origin of Abelian gauge group factors in heterotic/F-theory duality for a class of compactifications, the analysis provides a stage for further studies, both by extending the systematics of the analysis and by further detailed studies of the dual heterotic geometry and vector bundle data.

- It would be important to extend the studies to examples within larger classes of pairs of dual toric varieties as well as of more general elliptically fibered Calabi-Yau manifolds, respectively. In particular, this would allow to account for studies of dual geometries with broader classes of complex structure moduli spaces, and thus for an analysis of more general spectral covers of dual heterotic vector bundles. In $6 \mathrm{D}$ our analysis has been limited to a specific elliptically fibered Calabi-Yau $(n+1)$-fold, which has resulted in constrained appearances of non-Abelian gauge symmetries and additional U(1)'s. In particular, it would be illuminating to elaborate on the stable degeneration limit for general toric fibrations of two-dimensional polyhedra over $\mathbb{P}^{1}$ in $8 \mathrm{D}$ and, in addition, over Hirzebruch surfaces in $6 \mathrm{D}$. 
- It would be interesting to have the tools to study the spectral cover directly in the $\mathrm{Bl}_{1} \mathbb{P}^{(1,1,2)}$ model or more generally for fiber geometries which are given by the sixteen two-dimensioal reflexive polyhedra. This would require in particular a notion of the group law for these representations of elliptic curves.

- The study of the properties of the spectral cover was primarily confined to the derivation of the resulting gauge symmetries and the structure groups of the heterotic vector bundles. Further analysis of the spectral cover in compactifications to 6D (and extensions to 4D) is needed; it should shed light on the further spectral cover data, which enter Chern classes, anomaly cancellation and matter spectrum calculations. This study is complicated by the resolution of singularities of the heterotic geometry that may have to be performed, resulting in spectral covers, which are not finite [55].

- Our analysis has been primarily constrained to studies of Abelian gauge symmetries in the language of a perturbative heterotic dual. Although we have encountered spectral covers which seem to describe small instantons, i.e. non-perturbative M5branes, with discrete holonomy, we have not systematically analyzed their effect. In F-theory, M5-branes are visible as non-minimal singularities which occur at codimension two loci that have to be blown up. It would be interesting to thoroughly perform this geometric analysis. We expect in addition rich structures of Abelian gauge symmetry factors in F-theory whose heterotic duals are due to other types of non-perturbative M5-branes. Furthermore, it would be interesting to study the geometric transitions between F-theory geometries with different numbers of tensor multiplets, whose discussion is again related to this resolution process.

\section{Acknowledgments}

It is a pleasure to thank Hernan Piragua for initial collaboration at early stages of this project. We thank L. Anderson, R. Donagi, J. Gray, T. Grimm, A. Klemm, D. Morrison, R. Pardini, S. Schäfer-Nameki, V. Perduca and W. Taylor for useful discussions. We are grateful to the Theory Division of CERN (M.C. and M.P.), the 2015 Summer Program on "F-theory at the interface of particle physics and mathematics" at the Aspen Center for Physics (M.C., A.G., D.K. and M.P.) and the UPenn (D.K.) for hospitality during the course of the project. This research is supported in part by the DOE Grant Award DE-SC0013528, (M.C., M.P., P.S.), UPenn School of Arts and Sciences Funds for Faculty Working Group (A.G. and M.C.), the Fay R. and Eugene L. Langberg Endowed Chair (M.C.) and the Slovenian Research Agency (ARRS) (M.C.).

\section{A Weierstrass and Tate form of the hypersurface $\chi$}

In this appendix, we summarize the Weierstrass normal form as well as the Tate coefficients of the $\chi$ model. For convenience, we recall the most general form of the hypersurface $\chi$ 
which reads in the limit $x_{4} \rightarrow 1, x_{5} \rightarrow 1$

$\chi:=s_{1} x_{1}^{4}+s_{2} x_{1}^{3} x_{2}+s_{3} x_{1}^{2} x_{2}^{2}+s_{4} x_{1} x_{2}^{3}+s_{5} x_{1}^{2} x_{3}+s_{6} x_{1} x_{2} x_{3}+s_{7} x_{2}^{2} x_{3}+s_{8} x_{3}^{2}=0, \quad s_{i} \in \mathcal{O}_{\mathbb{P}^{1}}(2)$.

This can be brought in the so-called Tate form

$$
y^{2}+a_{1} x y+a_{3} y=x^{3}+a_{2}+a_{4} x+a_{6} .
$$

The Tate coefficients are explicitly given as [30]

$$
\begin{aligned}
& a_{1}=s_{6}, \\
& a_{2}=-s_{5} s_{7}-s_{3} s_{8}, \\
& a_{3}=-s_{4} s_{5} s_{8}-s_{2} s_{7} s_{8}, \\
& a_{4}=s_{3} s_{5} s_{7} s_{8}+s_{1} s_{7}^{2} s_{8}+s_{2} s_{4} s_{8}^{2}, \\
& a_{6}=-s_{1} s_{3} s_{7}^{2} s_{8}^{2}-s_{1} s_{4}^{2} s_{8}^{3}+s_{4} s_{7}\left(-s_{2} s_{5} s_{8}^{2}+s_{1} s_{6} s_{8}^{2}\right) .
\end{aligned}
$$

In addition, it is useful, to introduce the quantities

$$
\begin{aligned}
& b_{2}=a_{1}^{2}+4 a_{2}, \\
& b_{4}=a_{1} a_{3}+2 a_{4}, \\
& b_{6}=a_{3}^{2}+4 a_{6} .
\end{aligned}
$$

The Weierstrass normal form of $\chi^{\text {sing }}$ reads

$$
\begin{aligned}
f=( & -\frac{1}{48} s_{62}^{4}+\frac{1}{6} s_{52} s_{62}^{2} s_{72}-\frac{1}{3} s_{52}^{2} s_{72}^{2}-\frac{1}{2} s_{42} s_{52} s_{62} s_{82}+\frac{1}{6} s_{32} s_{62}^{2} s_{82} \\
& \left.+\frac{1}{3} s_{32} s_{52} s_{72} s_{82}-\frac{1}{2} s_{22} s_{62} s_{72} s_{82}+s_{21} s_{72}^{2} s_{82}-\frac{1}{3} s_{32}^{2} s_{82}^{2}+s_{22} s_{42} s_{82}^{2}\right) \\
g=( & \frac{1}{864} s_{62}^{6}-\frac{1}{72} s_{52} s_{62}^{4} s_{72}+\frac{1}{18} s_{52}^{2} s_{62}^{2} s_{72}^{2}-\frac{2}{27} s_{52}^{3} s_{72}^{3}+\frac{1}{24} s_{42} s_{52} s_{62}^{3} s_{82} \\
& -\frac{1}{72} s_{32} s_{62}^{4} s_{82}-\frac{1}{6} s_{42} s_{52}^{2} s_{62} s_{72} s_{82}+\frac{1}{36} s_{32} s_{52} s_{62}^{2} s_{72} s_{82}+\frac{1}{24} s_{22} s_{62}^{3} s_{72} s_{82} \\
& +\frac{1}{9} s_{32} s_{52}^{2} s_{72}^{2} s_{82}-\frac{1}{6} s_{22} s_{52} s_{62} s_{72}^{2} s_{82}-\frac{1}{12} s_{21} s_{62}^{2} s_{72}^{2} s_{82}+\frac{1}{3} s_{21} s_{52} s_{72}^{3} s_{82} \\
& +\frac{1}{4} s_{42}^{2} s_{52}^{2} s_{82}^{2}-\frac{1}{6} s_{32} s_{42} s_{52} s_{62} s_{82}^{2}+\frac{1}{18} s_{32}^{2} s_{62}^{2} s_{82}^{2}-\frac{1}{12} s_{22} s_{42} s_{62}^{2} s_{82}^{2} \\
& +\frac{1}{9} s_{32}^{2} s_{52} s_{72} s_{82}^{2}-\frac{1}{6} s_{22} s_{42} s_{52} s_{72} s_{82}^{2}-\frac{1}{6} s_{22} s_{32} s_{62} s_{72} s_{82}^{2}+s_{21} s_{42} s_{62} s_{72} s_{82}^{2} \\
& \left.+\frac{1}{4} s_{22}^{2} s_{72}^{2} s_{82}^{2}-\frac{2}{3} s_{21} s_{32} s_{72}^{2} s_{82}^{2}-\frac{2}{27} s_{32}^{3} s_{82}^{3}+\frac{1}{3} s_{22} s_{32} s_{42} s_{82}^{3}-s_{21} s_{42}^{2} s_{82}^{3}\right) .
\end{aligned}
$$

In particular, the discriminant reads

$$
\Delta=4 f^{3}+27 g^{2}=\frac{1}{48} s_{82}^{2}(\ldots) .
$$

where the expression in the bracket denotes a generic polynomial. 


\section{A.1 The map to Weierstrass normal form}

In this subsection we discuss the bi-rational map of (A.1) to Weierstrass normal form. As a first step, we transform (4.2) into the form

$$
\tilde{s}_{1} x_{1}^{4}+\tilde{s}_{2} x_{1}^{3} x_{2}+\tilde{s}_{3} x_{1}^{2} x_{2}^{2}+\tilde{s}_{4} x_{1} x_{2}^{3}+s_{7} x_{2}^{2} x_{3}+x_{3}^{2}=0 .
$$

Here, we have introduced the new quantities

$$
\tilde{s}_{1}=-\frac{1}{4} s_{5}^{2}+s_{0} s_{8}, \quad \tilde{s}_{2}=-\frac{1}{2} s_{5} s_{6}+s_{1} s_{8}, \quad \tilde{s}_{3}=-\frac{1}{4} s_{6}^{2}-\frac{1}{2} s_{5} s_{7}+s_{3} s_{8}, \quad \tilde{s}_{4}=-\frac{1}{2} s_{6} s_{7}+s_{4} s_{8}
$$

Next, one uses the transformations provided in [22]

$$
\begin{aligned}
x_{1} \longmapsto & z \\
x_{2} \longmapsto & \frac{6 s_{7} y+6 \tilde{s}_{4} x z+2 \tilde{s}_{3} \tilde{s}_{4} z^{3}+3 \tilde{s}_{2} s_{7}^{2} z^{3}}{2\left(3 s_{7}^{2} x-3 \tilde{s}_{4}^{2} z^{2}-2 \tilde{s}_{3} s_{7}^{2} z^{2}\right)} \\
x_{3} \longmapsto & \left(108 s_{7}^{3} x^{3}-108 s_{7}^{3} y^{2}-108 \tilde{s}_{4} s_{7}^{2} x y z-216 \tilde{s}_{4}^{2} s_{7} x^{2} z^{2}-108 \tilde{s}_{3} s_{7}^{3} x^{2} z^{2}-108 \tilde{s}_{4}^{3} y z^{3}\right. \\
& -144 \tilde{s}_{3} \tilde{s}_{4} s_{7}^{2} y z^{3}-108 \tilde{s}_{2} s_{7}^{4} y z^{3}-36 \tilde{s}_{3} \tilde{s}_{4}^{2} s_{7} x z^{4}-54 \tilde{s}_{2} \tilde{s}_{4} s_{7}^{3} x z^{4}+12 \tilde{s}_{3}^{2} \tilde{s}_{4}^{2} s_{7} z^{6} \\
& \left.-54 \tilde{s}_{2} \tilde{s}_{4}^{3} s_{7} z^{6}+16 \tilde{s}_{3}^{3} s_{7}^{3} z^{6}-72 \tilde{s}_{2} \tilde{s}_{3} \tilde{s}_{4} s_{7}^{3} z^{6}-27 \tilde{s}_{2}^{2} s_{7}^{5} z^{6}\right) / \\
& 12\left(3 s_{7}^{2} x-3 \tilde{s}_{4}^{2} z^{2}-2 \tilde{s}_{3} s_{7}^{2} z^{2}\right)^{2}
\end{aligned}
$$

in order to finally bring (A.7) into Weierstrass normal form in $\mathbb{P}^{(1,2,3)}$. We also note that the transformations (A.9) simplify in the case $s_{7}=0$, in particular their denominators loose their dependence on $x, y$.

\section{B Spectral cover examples with no U(1)}

For convenience and to demonstrate how our formalism works in a well-understood situation, we analyze several examples with pure non-Abelian gauge content only. These are related to the examples 4.2.1, 4.2.2 and 4.2.3 by a Higgsing process which gives $s_{72}$ a vacuum expectation value.

\section{B.1 Trivial structure group: $\mathrm{E}_{8} \times \mathrm{E}_{8}$ gauge symmetry}

As described in the section 4.1, we can obtain examples with higher rank gauge symmetry by specializing the coefficients of $\chi$. Aiming for a model with $\mathrm{E}_{8} \times \mathrm{E}_{8}$ gauge symmetry, one obtains the following coefficients.

\begin{tabular}{|c|c|c|c|}
\hline Coefficient & $\mathrm{K} 3$ & $X^{-}$ & $X^{+}$ \\
\hline$s_{1}$ & $s_{11} U^{2}+s_{12} U V+s_{13} V^{2}$ & $s_{12} U+s_{13} \mu$ & $s_{12} V+s_{11} \lambda_{1}$ \\
$s_{2}$ & $s_{22} U V$ & $s_{22} U$ & $s_{22} V$ \\
$s_{3}$ & $s_{32} U V$ & $s_{32} U$ & $s_{32} V$ \\
$s_{4}$ & $s_{42} U V$ & $s_{42} U$ & $s_{42} V$ \\
$s_{5}$ & $s_{52} U V$ & $s_{52} U$ & $s_{52} V$ \\
$s_{6}$ & $s_{62} U V$ & $s_{62} U$ & $s_{62} V$ \\
$s_{7}$ & 0 & 0 & 0 \\
$s_{8}$ & $s_{82} U V$ & $s_{82} U$ & $s_{82} V$ \\
\hline
\end{tabular}


Here the second row displays the coefficients of the full K3 surface while the coefficients of the two half K3 surfaces are displayed in row three and four. In particular, one notices that the coefficient $s_{7}$ is missing which means that one is passing from the toric ambient space $\mathrm{Bl}_{1} \mathbb{P}^{(1,1,2)} \times \mathbb{P}^{1}$ to the ambient space $\mathbb{P}^{(1,2,3)} \times \mathbb{P}^{1}$. Clearly, a generic section of the anti-canonical bundle of $\mathbb{P}^{(1,2,3)}$ does not have a second rational section, so there is also no reason to expect any $\mathrm{U}(1)$.

We proceed by analyzing the F-theory gauge group. The analysis of the Tate vectors reveals that

$$
\vec{t}_{U}=\vec{t}_{V}=(1,2,3,4,5,10)
$$

and thus there is an $\mathrm{E}_{8} \times \mathrm{E}_{8}$ gauge symmetry. After the stable degeneration limit, both half $\mathrm{K} 3$ surfaces $X^{+}$and $X^{-}$obtain one $\mathrm{E}_{8}$ singularity each.

Finally, we turn to the heterotic side. The splitting of the two half K3's into the heterotic elliptic curve and the spectral cover contributions reveals that

$$
p^{+}=s_{11} x_{1}^{4}, \quad p^{-}=s_{13} x_{1}^{4} .
$$

After transforming these expressions into the affine Weierstrass coordinates $x, y$, one obtains

$$
p_{W}^{+}=s_{11}, \quad p_{W}^{-}=s_{13}
$$

In both cases, one obtains an $\mathrm{SU}(1)$ spectral cover. However, the centralizer of the identity in $\mathrm{E}_{8}$ is $\mathrm{E}_{8}$ and one obtains a perfect match with the F-theory calculation.

\section{B.2 Structure group $\mathrm{SU}(1) \times \mathrm{SU}(2): \mathrm{E}_{8} \times \mathrm{E}_{7}$ gauge symmetry}

We consider the following model which is specified by the following coefficients in (4.2).

\begin{tabular}{|c|c|c|c|}
\hline Coefficient & $\mathrm{K} 3$ & $X^{-}$ & $X^{+}$ \\
\hline$s_{1}$ & $s_{11} U^{2}+s_{12} U V+s_{13} V^{2}$ & $s_{12} U+s_{13} \mu$ & $s_{12} V+s_{11} \lambda_{1}$ \\
$s_{2}$ & $s_{21} U^{2}+s_{22} U V$ & $s_{22} U$ & $s_{22} V+s_{21} \lambda_{1}$ \\
$s_{3}$ & $s_{32} U V$ & $s_{32} U$ & $s_{32} V$ \\
$s_{4}$ & $s_{42} U V$ & $s_{42} U$ & $s_{42} V$ \\
$s_{5}$ & $s_{52} U V$ & $s_{52} U$ & $s_{52} V$ \\
$s_{6}$ & $s_{62} U V$ & $s_{62} U$ & $s_{62} V$ \\
$s_{7}$ & 0 & 0 & 0 \\
$s_{8}$ & $s_{82} U V$ & $s_{82} U$ & $s_{82} V$ \\
\hline
\end{tabular}

This time, we obtain the following Tate vectors

$$
\vec{t}_{V}=(1,2,3,3,5,9), \quad \vec{t}_{U}=(1,2,3,4,5,10)
$$

which signal an $\mathrm{E}_{7}$ singularity at $V=0$ as well as an $\mathrm{E}_{8}$ singularity at $U=0$. The latter one is inherited by the half $\mathrm{K} 3$ surface $X^{-}$while the former one moves into $X^{+}$.

The spectral cover is in this case given by

$$
p^{+}=x_{1}^{3}\left(s_{11} x_{1}+s_{21} x_{2}\right), \quad p^{-}=s_{11} x_{1}^{4} .
$$


We only comment on the non-trivial spectral cover. After applying the transformation (A.9), it reads

$$
p_{W}^{+}=c_{0}+c_{1} x
$$

which defines an $\mathrm{SU}(2)$ spectral cover and is precisely what is expected. Explicitly, the $c_{i}$ 's read

$$
c_{0}=s_{21} s_{62}^{2}-4 s_{21} s_{32} s_{82}+12 s_{11} s_{42} s_{82} \quad c_{1}=-s_{21}
$$

Note that the $c_{i}$ are indeed proportional to $s_{11}, s_{21}$ which define the spectral cover. Thus, we obtain an $\mathrm{SU}(2)$ spectral cover in the case of $X^{+}$and a trivial structure group for the case of $X^{-}$. In conclusion, there is a perfect match with the F-theory analysis.

\section{B.3 Example with gauge group $\mathrm{E}_{8} \times \mathrm{SO}(11)$}

We consider the following model which is specified by the following coefficients in (4.2).

\begin{tabular}{|c|c|c|c|}
\hline Coefficient & $\mathrm{K} 3$ & $X^{-}$ & $X^{+}$ \\
\hline$s_{1}$ & $s_{11} U^{2}+s_{12} U V+s_{13} V^{2}$ & $s_{12} U+s_{13} \mu$ & $s_{12} V+s_{11} \lambda_{1}$ \\
$s_{2}$ & $s_{21} U^{2}+s_{22} U V$ & $s_{22} U$ & $s_{22} V+s_{21} \lambda_{1}$ \\
$s_{3}$ & $s_{31} U^{2}+s_{32} U V$ & $s_{32} U$ & $s_{32} V+s_{31} \lambda_{1}$ \\
$s_{4}$ & $s_{42} U V$ & $s_{42} U$ & $s_{42} V$ \\
$s_{5}$ & $s_{52} U V$ & $s_{52} U$ & $s_{52} V$ \\
$s_{6}$ & $s_{62} U V$ & $s_{62} U$ & $s_{62} V$ \\
$s_{7}$ & 0 & 0 & 0 \\
$s_{8}$ & $s_{82} U V$ & $s_{82} U$ & $s_{82} V$ \\
\hline
\end{tabular}

This time, we obtain the following Tate vectors

$$
\vec{t}_{V}=(1,1,3,3,5,8), \quad \vec{t}_{U}=(1,2,3,4,5,10)
$$

which signal an $\mathrm{SO}(11)$ singularity at $V=0$ as well as an $\mathrm{E}_{8}$ singularity at $U=0$. The former one is inherited by the half $\mathrm{K} 3$ surface $X^{+}$while the latter one moves into $X^{-}$.

The spectral cover is in this case given by

$$
p^{+}=x_{1}^{2}\left(s_{11} x_{1}^{2}+s_{21} x_{1} x_{2}+s_{31} x_{2}^{2}\right), \quad p^{-}=s_{11} x_{1}^{4} .
$$

We only comment on the non-trivial spectral cover. After applying the transformation (A.9), it reads

$$
p_{W}^{+}=c_{0}+c_{1} x+c_{2} x^{2}
$$

which defines an $\mathrm{Sp}(2) \cong \mathrm{SO}(5)$ spectral cover ${ }^{15}$ [7] and is precisely what is expected. Thus, we obtain an $\mathrm{Sp}(2)$ spectral cover in the case of $X^{+}$and a trivial structure group for the case of $X^{-}$. The commutant of $\mathrm{SO}(5)$ within $\mathrm{E}_{8}$ is given by $\mathrm{SO}(11)$.

\footnotetext{
${ }^{15}$ Sometimes, $\operatorname{SP}(N)$ is denoted by $\operatorname{SP}(2 N)$.
} 


\section{Tuned models without rational sections}

In this appendix we reproduce $[22,54]$ the following

Lemma C.1. The two sections denoted by $x_{1}=0$ and $x_{4}=0$ in (4.3) merge into a single section if and only if $s_{7}=0$ in (4.2). Furthermore, the single section is given by $\left[x_{1}: x_{2}: x_{3}: x_{4}: x_{5}\right]=[0: 1: 1: 0: 1]$.

Proof. Suppose the two sections $x_{1}=0$ and $x_{4}=0$ merge into a single section. Then this single section obeys both $x_{1}=0$ and $x_{4}=0$, everywhere. Thus the Stanley-Reisner ideal requires $x_{2} \neq 0, x_{3} \neq 0$ and $x_{5} \neq 0$ everywhere. Making use of the skaling relations (3.7) of the resolved space $\mathrm{Bl}_{1} \mathbb{P}^{(1,1,2)}$, one obtains that this section is indeed given by $\left[x_{1}: x_{2}\right.$ : $\left.x_{3}: x_{4}: x_{5}\right]=[0: 1: 1: 0: 1]$.

Suppose now that $s_{7}=0$. Setting $x_{1}$ in (4.2) to zero, results in the equation $s_{8} x_{3}^{2} x_{4}=0$. As $x_{3} \neq 0$ due to the Stanley Reisner ideal, $x_{4}$ has to vanish as well resulting in the merging of the two sections. Similarly, $x_{4}=0$ requires that $s_{4} x_{1} x_{2}^{3} x_{5}^{2}=0$. The Stanley Reisner ideal requires $x_{2}$ and $x_{5}$ to be non-vanishing. Thus, there is also in this case only one section given by $\left[x_{1}: x_{2}: x_{3}: x_{4}: x_{5}\right]=[0: 1: 1: 0: 1]$.

\section{Non-commutativity of the semi-stable degeneration limit and the map to Weierstrass form}

We illustrate the non-commutativity of the diagram 1 using the above example with gauge group $\mathrm{E}_{7} \times \mathrm{SO}(9) \times \mathrm{U}(1)$. To be precise, on the top left corner of the diagram, the section $\chi$ of $-K_{\mathrm{Bl}_{1} \mathbb{P}^{(1,1,2)} \times \mathbb{P}^{1}}$ is given by

$$
\chi: s_{1} x_{1}^{4}+s_{2} x_{1}^{3} x_{2}+s_{3} x_{1}^{2} x_{2}^{2}+s_{4} x_{1} x_{2}^{3}+s_{5} x_{1}^{2} x_{3}+s_{6} x_{1} x_{2} x_{3}+s_{7} x_{2}^{2} x_{3}+s_{8} x_{3}^{2}=0,
$$

where $s_{1}=s_{11} U^{2}+s_{12} U V+s_{13} V^{2}, \quad s_{i}=s_{i 1} U^{2}+s_{i 2} U V$ for $2 \leqslant i \leqslant 3$,

$$
s_{i}=s_{i 2} U V \text { for } 4 \leqslant i \leqslant 8 \text {. }
$$

Under the stable degeneration limit, denoted by the left map in the diagram $1, \chi$ is split into $\chi^{ \pm}$, which are in turn defined by

$$
\begin{aligned}
\chi^{ \pm}: & s_{1}^{ \pm} x_{1}^{4}+s_{2}^{ \pm} x_{1}^{3} x_{2}+s_{3}^{ \pm} x_{1}^{2} x_{2}^{2}+s_{4}^{ \pm} x_{1} x_{2}^{3}+s_{5}^{ \pm} x_{1}^{2} x_{3}+s_{6}^{ \pm} x_{1} x_{2} x_{3}+s_{7}^{ \pm} x_{2}^{2} x_{3}+s_{8}^{ \pm} x_{3}^{2}=0, \\
\text { where } & s_{1}^{+}=s_{12} U+s_{13} \mu, \quad s_{1}^{-}=s_{11} \lambda_{1}+s_{12} V, \\
& s_{i}^{+}=s_{i 2} U \text { and } s_{i}^{-}=s_{i 1} \lambda_{1}+s_{i 2} V \text { for } 2 \leqslant i \leqslant 3 \\
& s_{i}^{+}=s_{i 2} U \text { and } s_{i}^{-}=s_{i 2} V \text { for } 4 \leqslant i \leqslant 8 .
\end{aligned}
$$

We further map $\chi^{ \pm}$, under the bottom map of the diagram 1, into their respective Weierstrass forms

$$
W_{\chi}^{ \pm}: y^{2}=x^{3}+f_{\chi}^{ \pm} x z^{4}+g_{\chi}^{ \pm} z^{6} .
$$

We can show that $W_{\chi}^{ \pm}$obtained in this way is different compared to $W_{\chi}^{\prime} \pm$ obtained by taking the other route in diagram 1, namely start from $\chi$ on the top left corner of the 
diagram, first map $\chi$ into its Weierstrass form $W_{\chi}$ using the map on top of 1 , and then use the map on the right of 1 to split $W_{\chi}$ into

$$
W_{\chi}^{\prime \pm}: y^{2}=x^{3}+f_{\chi}^{\prime \pm} x z^{4}+g_{\chi}^{\prime \pm} z^{6} .
$$

Indeed,

$$
W_{\chi}^{+} \neq W_{\chi}^{+}, \quad W_{\chi}^{-} \neq W_{\chi}^{\prime-}
$$

To be precise,

$f_{\chi}^{ \pm}=f_{\chi}^{\prime \pm} \quad$ but $\quad g_{\chi}^{ \pm} \neq g_{\chi}^{\prime}, \quad g_{\chi}^{+}-g_{\chi}^{\prime+}=\frac{2}{3} U^{6} s_{13} s_{31} s_{72}^{2} s_{82}^{2}, \quad g_{\chi}^{-}-g_{\chi}^{\prime}=\frac{2}{3} V^{6} s_{13} s_{31} s_{72}^{2} s_{82}^{2}$.

Open Access. This article is distributed under the terms of the Creative Commons Attribution License (CC-BY 4.0), which permits any use, distribution and reproduction in any medium, provided the original author(s) and source are credited.

\section{References}

[1] M.B. Green, J.H. Schwarz and E. Witten, Superstring theory. Volume 2: Loop amplitudes, anomalies and phenomenology, Cambridge University Press, Cambridge U.K. (2012).

[2] J. Polchinski, String theory. Volume 1: An introduction to the bosonic string. Volume 2: Superstring theory and beyond, Cambridge University Press, Cambridge U.K. (1998).

[3] C. Vafa, Evidence for F-theory, Nucl. Phys. B 469 (1996) 403 [hep-th/9602022] [InSPIRE].

[4] D.R. Morrison and C. Vafa, Compactifications of F-theory on Calabi-Yau threefolds (II), Nucl. Phys. B 476 (1996) 437 [hep-th/9603161] [INSPIRE].

[5] D.R. Morrison and C. Vafa, Compactifications of F-theory on Calabi-Yau threefolds (I), Nucl. Phys. B 473 (1996) 74 [hep-th/9602114] [INSPIRE].

[6] P.S. Aspinwall and D.R. Morrison, Point-like instantons on K3 orbifolds, Nucl. Phys. B 503 (1997) 533 [hep-th/9705104] [INSPIRE].

[7] R. Friedman, J. Morgan and E. Witten, Vector bundles and F-theory, Commun. Math. Phys. 187 (1997) 679 [hep-th/9701162] [INSPIRE].

[8] R.Y. Donagi, Principal bundles on elliptic fibrations, Asian J. Math. 1 (1997) 214 [alg-geom/9702002] [INSPIRE].

[9] B. Andreas, $N=1$ heterotic/F theory duality, Fortschr. Phys. 47 (1999) 587 [hep-th/9808159] [INSPIRE].

[10] P. Candelas and A. Font, Duality between the webs of heterotic and type-II vacua, Nucl. Phys. B 511 (1998) 295 [hep-th/9603170] [INSPIRE].

[11] P. Berglund and P. Mayr, Heterotic string/F-theory duality from mirror symmetry, Adv. Theor. Math. Phys. 2 (1999) 1307 [hep-th/9811217] [INSPIRE].

[12] L.B. Anderson and W. Taylor, Geometric constraints in dual F-theory and heterotic string compactifications, JHEP 08 (2014) 025 [arXiv: 1405.2074] [INSPIRE].

[13] H. Hayashi, R. Tatar, Y. Toda, T. Watari and M. Yamazaki, New aspects of heterotic-F-theory duality, Nucl. Phys. B 806 (2009) 224 [arXiv: 0805.1057] [INSPIRE]. 
[14] T.W. Grimm, T.-W. Ha, A. Klemm and D. Klevers, Computing brane and flux superpotentials in F-theory compactifications, JHEP 04 (2010) 015 [arXiv:0909.2025] [INSPIRE].

[15] T.W. Grimm, T.-W. Ha, A. Klemm and D. Klevers, Five-brane superpotentials and heterotic/F-theory duality, Nucl. Phys. B 838 (2010) 458 [arXiv:0912.3250] [INSPIRE].

[16] H. Jockers, P. Mayr and J. Walcher, On $\mathcal{N}=14$ effective couplings for F-theory and heterotic vacua, Adv. Theor. Math. Phys. 14 (2010) 1433 [arXiv:0912.3265] [InSPIRE].

[17] M. Cvetič, I. García Etxebarria and J. Halverson, Three looks at instantons in F-theory new insights from anomaly inflow, string junctions and heterotic duality, JHEP 11 (2011) 101 [arXiv:1107.2388] [INSPIRE].

[18] M. Cvetič, R. Donagi, J. Halverson and J. Marsano, On seven-brane dependent instanton prefactors in F-theory, JHEP 11 (2012) 004 [arXiv: 1209.4906] [INSPIRE].

[19] G. Aldazabal, A. Font, L.E. Ibáñez and A.M. Uranga, New branches of string compactifications and their F-theory duals, Nucl. Phys. B 492 (1997) 119 [hep-th/9607121] [INSPIRE].

[20] A. Klemm, P. Mayr and C. Vafa, BPS states of exceptional noncritical strings, hep-th/9607139 [INSPIRE].

[21] A. Grassi, Toric Weierstrass models, talk given at the Workshop on Elliptic fibrations and F-theory, Kavli I.P.M.U., Tokyo Japan (2010), http://www.ipmu.jp/node/552.

[22] D.R. Morrison and D.S. Park, F-theory and the Mordell-Weil group of elliptically-fibered Calabi-Yau threefolds, JHEP 10 (2012) 128 [arXiv:1208.2695] [INSPIRE].

[23] J. Borchmann, C. Mayrhofer, E. Palti and T. Weigand, Elliptic fibrations for $\mathrm{SU}(5) \times \mathrm{U}(1) \times \mathrm{U}(1)$ F-theory vacua, Phys. Rev. D 88 (2013) 046005 [arXiv:1303.5054] [INSPIRE].

[24] M. Cvetič, D. Klevers and H. Piragua, F-theory compactifications with multiple U(1)-factors: constructing elliptic fibrations with rational sections, JHEP 06 (2013) 067 [arXiv:1303.6970] [INSPIRE].

[25] M. Cvetič, A. Grassi, D. Klevers and H. Piragua, Chiral four-dimensional F-theory compactifications with $\mathrm{SU}(5)$ and multiple $\mathrm{U}(1)$-factors, JHEP 04 (2014) 010 [arXiv: 1306.3987] [INSPIRE].

[26] J. Borchmann, C. Mayrhofer, E. Palti and T. Weigand, SU(5) tops with multiple U(1)s in F-theory, Nucl. Phys. B 882 (2014) 1 [arXiv:1307.2902] [INSPIRE].

[27] M. Cvetič, D. Klevers and H. Piragua, F-theory compactifications with multiple U(1)-factors: addendum, JHEP 12 (2013) 056 [arXiv:1307.6425] [INSPIRE].

[28] M. Cvetič, D. Klevers, H. Piragua and P. Song, Elliptic fibrations with rank three Mordell-Weil group: F-theory with $\mathrm{U}(1) \times \mathrm{U}(1) \times \mathrm{U}(1)$ gauge symmetry, JHEP 03 (2014) 021 [arXiv: 1310.0463] [INSPIRE].

[29] V. Braun, T.W. Grimm and J. Keitel, Complete intersection fibers in F-theory, JHEP 03 (2015) 125 [arXiv: 1411.2615] [INSPIRE].

[30] D. Klevers, D.K. Mayorga Pena, P.-K. Oehlmann, H. Piragua and J. Reuter, F-theory on all toric hypersurface fibrations and its Higgs branches, JHEP 01 (2015) 142 [arXiv:1408.4808] [INSPIRE]. 
[31] M. Cvetič, D. Klevers, H. Piragua and W. Taylor, General U(1) $\times$ U(1) F-theory compactifications and beyond: geometry of unHiggsings and novel matter structure, JHEP 11 (2015) 204 [arXiv:1507.05954] [INSPIRE].

[32] A. Grassi and V. Perduca, Weierstrass models of elliptic toric K3 hypersurfaces and symplectic cuts, Adv. Theor. Math. Phys. 17 (2013) 741 [arXiv:1201.0930] [InSPIRE].

[33] R. Blumenhagen, G. Honecker and T. Weigand, Loop-corrected compactifications of the heterotic string with line bundles, JHEP 06 (2005) 020 [hep-th/0504232] [INSPIRE].

[34] J. Marsano, N. Saulina and S. Schäfer-Nameki, Monodromies, fluxes and compact three-generation F-theory GUTs, JHEP 08 (2009) 046 [arXiv:0906.4672] [INSPIRE].

[35] R. Blumenhagen, T.W. Grimm, B. Jurke and T. Weigand, Global F-theory GUTs, Nucl. Phys. B 829 (2010) 325 [arXiv:0908.1784] [InSPIRE].

[36] T.W. Grimm and T. Weigand, On Abelian gauge symmetries and proton decay in global F-theory GUTs, Phys. Rev. D 82 (2010) 086009 [arXiv: 1006.0226] [INSPIRE].

[37] P.S. Aspinwall and D.R. Morrison, Nonsimply connected gauge groups and rational points on elliptic curves, JHEP 07 (1998) 012 [hep-th/9805206] [INSPIRE].

[38] K.-S. Choi and H. Hayashi, U(n) spectral covers from decomposition, JHEP 06 (2012) 009 [arXiv: 1203.3812] [INSPIRE].

[39] P.S. Aspinwall, K3 surfaces and string duality, in Fields, strings and duality. Proceedings, Summer School, Theoretical Advanced Study Institute in Elementary Particle Physics, TASI'96, Boulder U.S.A., 2-28 Jun 1996, pp. 421-540 [hep-th/9611137] [INSPIRE].

[40] C. Vafa and E. Witten, Dual string pairs with $N=1$ and $N=2$ supersymmetry in four-dimensions, Nucl. Phys. Proc. Suppl. 46 (1996) 225 [hep-th/9507050] [InSPIRE].

[41] M. Bershadsky et al., Geometric singularities and enhanced gauge symmetries, Nucl. Phys. B 481 (1996) 215 [hep-th/9605200] [INSPIRE].

[42] P.S. Aspinwall, Aspects of the hypermultiplet moduli space in string duality, JHEP 04 (1998) 019 [hep-th/9802194] [INSPIRE].

[43] K. Kodaira, On compact analytic surfaces II, Ann. Math. 77 (1963) 563.

[44] M.R. Douglas, D.S. Park and C. Schnell, The Cremmer-Scherk mechanism in F-theory compactifications on K3 manifolds, JHEP 05 (2014) 135 [arXiv: 1403.1595] [INSPIRE].

[45] R. Donagi, ICMP lecture on heterotic/F-theory duality, in Mathematical physics. Proceedings, 12th International Congress, ICMP'g7, Brisbane Australia, 13-19 Jul 1997 [hep-th/9802093] [INSPIRE].

[46] M.F. Atiyah, Vector bundles over an elliptic curve, Proc. London Math. Soc. s3-7 (1957) 414.

[47] R. Friedman, J.W. Morgan and E. Witten, Vector bundles over elliptic fibrations, alg-geom/9709029 [INSPIRE].

[48] J.W. Morgan, Holomorphic bundles over elliptic manifolds, lecture given at the School on Algebraic Geometry, Trieste Italy, 26 Jul-13 Aug 1999.

[49] P.S. Aspinwall, An analysis of fluxes by duality, hep-th/0504036 [INSPIRE].

[50] J. Tate, Algorithm for determining the type of a singular fiber in an elliptic pencil, in Modular functions of one variable IV, Lect. Notes Math. 476 (1975) 33. 
[51] S. Katz, D.R. Morrison, S. Schäfer-Nameki and J. Sully, Tate's algorithm and F-theory, JHEP 08 (2011) 094 [arXiv: 1106.3854] [INSPIRE].

[52] U. Bruzzo and A. Grassi, Picard group of hypersurfaces in toric 3-folds, Int. J. Math. 23 (2012) 1250028 [arXiv:1011.1003].

[53] V.V. Batyrev, Dual polyhedra and mirror symmetry for Calabi-Yau hypersurfaces in toric varieties, J. Alg. Geom. 3 (1994) 493 [alg-geom/9310003] [INSPIRE].

[54] D.R. Morrison and W. Taylor, Sections, multisections and U(1) fields in F-theory, arXiv: 1404.1527 [INSPIRE].

[55] R. Donagi, B.A. Ovrut, T. Pantev and D. Waldram, Standard models from heterotic M-theory, Adv. Theor. Math. Phys. 5 (2002) 93 [hep-th/9912208] [INSPIRE].

[56] T. Shioda and K. Oguiso, The Mordell-Weil lattice of a rational elliptic surface, Comment. Math. Univ. St. Paul 40 (1991) 83.

[57] I. Shimada, On elliptic K3 surfaces, Michigan Math. J. 47 (2000) 423.

[58] C. Mayrhofer, D.R. Morrison, O. Till and T. Weigand, Mordell-Weil torsion and the global structure of gauge groups in F-theory, JHEP 10 (2014) 016 [arXiv:1405.3656] [INSPIRE].

[59] A. Grassi and D.R. Morrison, Anomalies and the Euler characteristic of elliptic Calabi-Yau threefolds, Commun. Num. Theor. Phys. 6 (2012) 51 [arXiv:1109.0042] [inSPIRE]. 